\title{
Susceptibilities and the Phase Structure of a Chiral Model with Polyakov Loops
}

\author{
C. Sasaki, ${ }^{1}$ B. Friman, ${ }^{1}$ and K. Redlich ${ }^{2}$ \\ ${ }^{1}$ Gesellschaft für Schwerionenforschung, GSI, D-64291 Darmstadt, Germany \\ ${ }^{2}$ Institute of Theoretical Physics, University of Wroclaw, PL-50204 Wroctaw, Poland
}

(Dated: October 2, 2018)

\begin{abstract}
In an extension of the Nambu-Jona-Lasinio model where the quarks interact with the temporal gluon field, represented by the Polyakov loop, we explore the relation between the deconfinement and chiral phase transitions. The effect of Polyakov loop dynamics on thermodynamic quantities, on the phase structure at finite temperature and baryon density and on various susceptibilities is presented. Particular emphasis is put on the behavior and properties of the fluctuations of the (approximate) order parameters and their dependence on temperature and net-quark number density. We also discuss how the phase structure of the model is influenced by the coupling of the quarks to the Polyakov loop.

PACS numbers: 25.75.Nq, 12.39.Fe
\end{abstract}

\section{INTRODUCTION}

Quantum Chromodynamics (QCD) exhibits dynamical chiral symmetry breaking and confinement. Both features are related with global symmetries of the QCD Lagrangian. However, the relation of spontaneous chiral symmetry breaking and confinement still remains an open issue. While the chiral symmetry is exact in the limit of massless quarks the $Z(3)$ center symmetry, which governs confinement, is exact in the opposite limit, i.e., for infinitely heavy quarks. In order to obtain a unified picture of confinement and chiral symmetry breaking several effective chiral models have been studied [1, 2, 3, , 4, 5, 6, 7, 8, 9]. Recently an extension of the Nambu-Jona-Lasinio (NJL) model 10, 11] was proposed and developed [2, 3, 5, 12, 13, 14, 15] to address this question.

The NJL model describes interactions of constituent quark fields. It exhibits a global SU(3) symmetry that is a replacement of a local gauge $\mathrm{SU}(3)$ color transformation of the QCD Lagrangian. Thus, color confinement is lost in the NJL dynamics. Recently, color degrees of freedom were introduced in the NJL Lagrangian through an effective gluon potential expressed in terms of Polyakov loops 2, 3, 5]. Such a potential was constructed to preserve the $Z(3)$ symmetry of the gluon part of the QCD Lagrangian and it has its origin in the recently developed effective models with Polyakov loops as dynamical fields 16]. An extended NJL Lagrangian (PNJL model) contains unified properties of QCD related with $Z(3)$ and the chiral symmetries. The interactions of quarks with the effective gluon fields in the PNJL model is included through covariant derivatives. Furthermore, due to the symmetries of the Lagrangian, the PNJL model belongs to the same universality class as that expected for QCD. Thus, such a model can be considered as a testing ground for studying the phase structure and critical phenomena related with the deconfinement and chiral phase transitions. This is particularly interesting since there are still limitations in the applicability of lattice gauge theory (LGT) to QCD thermodynamics at large net quark densities.

Recently, it was shown [5, 12, 13, 14] that the PNJL model, formulated at finite temperature and finite quark chemical potential, reproduces some of the thermodynamical observables computed within LGT. The properties of the equation of state [5], the in-medium modification of meson masses [15] as well as the validity and applicability of the Taylor expansion in quark chemical potential used in LGT were recently addressed within the PNJL model [12, 13, 14]. In Ref. 17] the model was extended to a system with finite isospin chemical potential and pion condensation was studied.

In this paper we will consider the fluctuations in various channels at points in the phase diagram near the phase boundary. In particular, we focus on the behavior of the net-quark number fluctuations and the susceptibilities of the order parameters ${ }^{\# 1}$. The susceptibilities of the Polyakov loop and its conjugate as well as the chiral condensate will be introduced and analyzed. The relation between the chiral and deconfinement phase transitions will be quantified through the susceptibilities. Our calculations are performed within the mean field approximation and in the PNJL model, including a non-local four-fermion interactions to regulate the divergent momentum integrals.

The paper is organized as follows: In Section 2 the PNJL model is formulated and the relevant thermodynamic potentials are derived. In Section 3 the influence of the Polyakov loop dynamics on various thermodynamical quantities is discussed. In Section 4 and Section 5 we introduce the susceptibilities of the order parameters

\footnotetext{
\#1 We will be somewhat lax, and refer to the Polyakov loop and the quark condensate as order parameters, although, due to the explicit breaking of the symmetries, neither of them is strictly speaking an order parameter. However, as found in LGT calculations, both are useful quantities for identifying the location of the phase boundary. Thus, it seems that the explicit breaking of both the chiral and center symmetry in QCD is in this sense weak.
} 
and focus on their properties. Concluding remarks and discussion are presented in Section 6 .

\section{TWO-FLAVOR NJL MODEL WITH THE POLYAKOV LOOP}

Various methods have been proposed to account for interactions with the color gauge field in effective chiral models [1, 2, 3, 5, 6, 7]. One of these is the extension of the Nambu-Jona-Lasinio (NJL) Lagrangian by coupling the quarks to a uniform temporal background gauge field, which manifests itself entirely in the Polyakov loop [2, 3, 5]. The PNJL Lagrangian for three colors $\left(N_{c}=3\right)$ and two flavors $\left(N_{f}=2\right)$ with non-local four-fermion interactions is given by

$$
\begin{aligned}
\mathcal{L}= & \bar{\psi}(i \not D-\hat{m}) \psi+\bar{\psi} \hat{\mu} \gamma_{0} \psi-\mathcal{U}(\Phi[A], \bar{\Phi}[A] ; T) \\
& +\frac{G_{S}}{2}\left[(\bar{q}(x) q(x))^{2}+\left(\bar{q}(x) i \gamma_{5} \vec{\tau} q(x)\right)^{2}\right]
\end{aligned}
$$

where $\hat{m}=\operatorname{diag}\left(m_{u}, m_{d}\right)$ is the current quark mass, $\hat{\mu}=$ $\operatorname{diag}\left(\mu_{u}, \mu_{d}\right)$ is the quark chemical potential and $\vec{\tau}$ are the Pauli matrices. We assume isospin symmetry and take $m_{u}=m_{d} \equiv m_{0}$ and $\mu_{u}=\mu_{d} \equiv \mu$.

The Lagrangian is formulated with non-local interactions, which are controlled by a form factor. This feature of the model is implemented in order to deal with the ultraviolet singularities that appear in the loop integrations. In coordinate space, the form factor $F(x)$ for the non-local current-current interaction reads:

$$
q(x)=\int d^{4} y F(x-y) \psi(y) .
$$

A possible choice for the regulator is in momentum space given by [18]:

$$
f^{2}(p)=\frac{1}{1+(p / \Lambda)^{2 \alpha}}
$$

where $f(p)$ is the Fourier transform of the form factor $F(x)$ and $p$ is the three-momentum.

The strength of the interaction among constituent quarks in (2.1) is parameterized by the coupling constant $G_{S}$ which carries the dimension of a length squared. In the pure NJL sector, the model is controlled by four parameters: the coupling constant $G_{S}$, the current quark mass $m_{0}$ and the constants $\alpha$ and $\Lambda$, which characterize the range of the non-locality. These parameters are determined in vacuum, for a given $\alpha$, by requiring that the experimental values of the pion decay constant $f_{\pi}=92.4$ $\mathrm{MeV}$ and the pion mass $m_{\pi}=135 \mathrm{MeV}$ as well as the dynamical quark mass $M_{p=0}=335 \mathrm{MeV}$ are reproduced. For $\alpha=10$ the model parameters are $\Lambda=684.2 \mathrm{MeV}$, $G_{S} \Lambda^{2}=4.66, m_{0}=4.46 \mathrm{MeV}$. The corresponding value of the quark condensate is $\langle\bar{\psi} \psi\rangle^{1 / 3}=-256.2 \mathrm{MeV}$ [19]. The relevant parameters of the model used in our calculations are summarized in Table @
The interaction between the effective gluon field and the quarks is in the PNJL Lagrangian implemented (2.1) by means of a covariant derivative

$$
D_{\mu}=\partial_{\mu}-i A_{\mu}, \quad A_{\mu}=\delta_{\mu 0} A^{0},
$$

where we introduce the standard short-hand notation $A_{\mu}=g A_{\mu}^{a} \frac{\lambda^{a}}{2}$. Here $g$ is the color $\mathrm{SU}(3)$ gauge coupling constant and $\lambda^{a}$ are the Gell-Mann matrices.

The effective potential $\mathcal{U}$ of the gluon field in (2.1) is expressed in terms of the traced Polyakov loop $\Phi$ and its conjugate $\bar{\Phi}$

$$
\Phi=\frac{1}{N_{c}} \operatorname{Tr}_{c} L, \quad \bar{\Phi}=\frac{1}{N_{c}} \operatorname{Tr}_{c} L^{\dagger},
$$

where $L$ is a matrix in color space related to the gauge field by

$$
L(\vec{x})=\mathcal{P} \exp \left[i \int_{0}^{\beta} d \tau A_{4}(\vec{x}, \tau)\right],
$$

with $\mathcal{P}$ being the path (Euclidean time) ordering, and $\beta=1 / T$ with $A_{4}=i A_{0}$.

In the heavy quark mass limit QCD has the Z(3) center symmetry which is spontaneously broken in the hightemperature phase. The thermal expectation value of the Polyakov loop $\langle\Phi\rangle$ acts as an order parameter of the $\mathrm{Z}(3)$ symmetry. Consequently, $\langle\Phi\rangle=0$ at low temperatures in the confined phase and $\langle\Phi\rangle \neq 0$ at high temperatures corresponding to the deconfined phase. For the $\mathrm{SU}_{c}(3)$ color gauge group the Polyakov loop matrix $L$ satisfies $L L^{\dagger}=1, \operatorname{det} L=1$ and can be written in diagonal form

$$
L=\operatorname{diag}\left(e^{i \varphi}, e^{i \varphi^{\prime}}, e^{-i\left(\varphi+\varphi^{\prime}\right)}\right) .
$$

In general $\Phi$ in Eq. 2.5 is not identical to $\bar{\Phi}$.

The effective potential $\mathcal{U}(\Phi, \bar{\Phi})$ of the gluon field is expressed in terms of the Polyakov loops so as to preserve the $Z(3)$ symmetry of the pure gauge theory [16]. We adopt an effective potential of the following form [5] \#2.

$$
\frac{\mathcal{U}(\Phi, \bar{\Phi} ; T)}{T^{4}}=-\frac{b_{2}(T)}{2} \bar{\Phi} \Phi-\frac{b_{3}}{6}\left(\Phi^{3}+\bar{\Phi}^{3}\right)+\frac{b_{4}}{4}(\bar{\Phi} \Phi)^{2},
$$

with

$$
b_{2}(T)=a_{0}+a_{1}\left(\frac{T_{0}}{T}\right)+a_{2}\left(\frac{T_{0}}{T}\right)^{2}+a_{3}\left(\frac{T_{0}}{T}\right)^{3} .
$$

\footnotetext{
\#2 The presence of quarks will clearly affect the Polyakov loop dynamics. Thus, one may ask why this is not reflected in the effective potential $\mathcal{U}$. However, at least partly such effects are treated explicitly in the PNJL model through the coupling to the quarks. Thus, in order to minimize the double counting problem, we keep the $Z(3)$ symmetric form of the effective potential, but consider the possibility that the parameters, in particular $T_{0}$, may depend on the number of dynamical flavors $N_{f}$, thus accounting for quark loop effects not included in the mean-field approximation.
} 
The coefficients $T_{0}, a_{i}$ and $b_{i}$ are fixed by requiring that the equation of state obtained in pure gauge theory on the lattice is reproduced. In particular, at $T_{0}=270 \mathrm{MeV}$ the model reproduces the first order deconfinement phase transition of the pure gauge theory. The parameters are listed in Table II.

In the mean field approximation the Lagrangian (2.1) is rewritten as

$$
\begin{aligned}
\mathcal{L}= & \bar{\psi}\left(i \not D+\hat{\mu} \gamma_{0}\right) \psi-\int d^{4} y_{1} d^{4} y_{2} \bar{\psi}\left(y_{1}\right) M\left(y_{1}, y_{2}, x\right) \psi\left(y_{2}\right) \\
& -\frac{G_{S}}{2}(\langle\bar{q}(x) q(x)\rangle)^{2}-\mathcal{U}(\Phi, \bar{\Phi} ; T)
\end{aligned}
$$

where

$$
\begin{aligned}
& M\left(y_{1}, y_{2}, x\right)=m_{0} \delta^{(4)}\left(x-y_{1}\right) \delta^{(4)}\left(x-y_{1}\right) \\
& +F\left(x-y_{1}\right) F\left(x-y_{2}\right)\langle\bar{q}(x) q(x)\rangle .
\end{aligned}
$$

In momentum space the dynamical quark mass is determined by the current quark mass $m_{0}$, the quark momentum distribution function $f(p)$ and the chiral condensate $\langle\bar{q} q\rangle$

$$
\begin{aligned}
& M_{p}=m_{0}+\left(M-m_{0}\right) f^{2}(p), \\
& M=m_{0}-G_{S}\langle\bar{q} q\rangle,
\end{aligned}
$$

where $M$ denotes the dynamical quark mass at $p=0$. In a uniform system, the chiral condensate

$$
\langle\bar{q} q\rangle=\int d^{4} p f(p)^{2}\langle\bar{\psi}(p) \psi(p)\rangle
$$

is independent of the position.

From the mean field Lagrangian (2.10) one obtains the thermodynamic potential in the following form

$$
\begin{aligned}
& \Omega(M, \Phi, \bar{\Phi} ; T, \mu)=\mathcal{U}(\Phi, \bar{\Phi} ; T)+\frac{\left(m_{0}-M\right)^{2}}{2 G_{S}} \\
& -6 N_{f} \int \frac{d^{3} p}{(2 \pi)^{3}}\left[E_{p}-E_{p}\left(M_{p}=m_{0}\right)\right] \\
& -2 N_{f} T \int \frac{d^{3} p}{(2 \pi)^{3}}\left\{\operatorname{Tr}_{c} \ln \left[1+L e^{-E^{(+)} / T}\right]\right. \\
& \left.+\operatorname{Tr}_{c} \ln \left[1+L^{\dagger} e^{-E^{(-)} / T}\right]\right\},
\end{aligned}
$$

where we have introduced $E^{( \pm)}=E_{p} \mp \mu$ for the particle $(+)$ and anti-particle $(-)$ with $E_{p}=\sqrt{|\vec{p}|^{2}+M_{p}^{2}}$ being a quasiparticle energy. The third term in the thermodynamic potential (2.14), which corresponds to the vacuum contribution, is renormalized by subtracting the term $E_{p}\left(M_{p}=m_{0}\right)$ under the momentum integral. This removes the divergence from and introduces an irrelevant constant shift of $\Omega$.

Furthermore, by taking the trace in color space, one obtains the final expression for the thermodynamic potential

$$
\begin{aligned}
& \Omega(M, \Phi, \bar{\Phi} ; T, \mu)=\mathcal{U}(\Phi, \bar{\Phi} ; T)+\frac{\left(m_{0}-M\right)^{2}}{2 G_{S}} \\
& -6 N_{f} \int \frac{d^{3} p}{(2 \pi)^{3}}\left[E_{p}-E_{p}\left(M_{p}=m_{0}\right)\right] \\
& -2 N_{f} T \int \frac{d^{3} p}{(2 \pi)^{3}}\left\{\ln \left[g^{(+)}(M, \Phi, \bar{\Phi} ; T, \mu ; p)\right]\right. \\
& \left.\quad+\ln \left[g^{(-)}(M, \Phi, \bar{\Phi} ; T, \mu ; p)\right]\right\}
\end{aligned}
$$

where

$$
\begin{aligned}
& g^{(+)}(M, \Phi, \bar{\Phi} ; T, \mu ; p) \\
& =1+3\left(\Phi+\bar{\Phi} e^{-E^{(+)} / T}\right) e^{-E^{(+)} / T}+e^{-3 E^{(+)} / T}, \\
& g^{(-)}(M, \Phi, \bar{\Phi} ; T, \mu ; p) \\
& =1+3\left(\bar{\Phi}+\Phi e^{-E^{(-)} / T}\right) e^{-E^{(-)} / T}+e^{-3 E^{(-)} / T} .
\end{aligned}
$$

Under the transformation $\mu \rightarrow-\mu$, the role of quarks and antiquarks is exchanged. Inspection of (2.16) shows that in the PNJL model the charge conjugation transformation also exchanges the role of the Polyakov loop and its conjugate. This is reflected in the relation $g^{(+)}(M, \Phi, \bar{\Phi} ; T, \mu ; p)=g^{(-)}(M, \bar{\Phi}, \Phi ; T,-\mu ; p)$.

Although the Polyakov loop is complex, the thermodynamic potential is real. Due to the symmetry in color space, the imaginary part of $\Omega$ vanishes after performing the functional integral [see Appendix $\mathrm{A}$ ].

An interesting feature of the PNJL model described by the thermodynamic potential (2.15) is the qualitative behavior in the low temperature phase. In the limit of $\Phi, \bar{\Phi} \rightarrow 0$, which is expected at low temperatures, the contribution of one- and two-quark states to $g^{( \pm)}$are suppressed and only the three-quark term $\sim \exp \left(-3 E^{( \pm)} / T\right)$ survives. In this sense the PNJL model mimics the confinement of quarks within threequark states. The suppression of quark degrees of freedom at low temperatures is, on a qualitative level, similar to confinement in QCD thermodynamics. Thus, the PNJL model is better suited for describing the low temperature QCD phase than the standard NJL model, where the constituent quarks are abundant also at low temperatures. However, at least in the mean-field approximation, the model only has the three-quark states, but there are no one- and two-quark states, which also play an important role at low temperatures.

In the mean field approximation the dynamical quark mass $M$ and the expectation values of the Polyakov loop $\Phi$ and $\bar{\Phi}$ are obtained from the stationarity conditions

$$
\frac{\partial \Omega}{\partial M}=\frac{\partial \Omega}{\partial \Phi}=\frac{\partial \Omega}{\partial \bar{\Phi}}=0,
$$

obtained by extremizing the thermodynamic potential with respect to $M, \Phi$ and $\bar{\Phi}$. 
These conditions yield the following set of coupled gap equations:

$$
\begin{aligned}
& M=m_{0}+6 G_{S} N_{f} \int \frac{d^{3} p}{(2 \pi)^{3}} \frac{M_{p} f^{2}(p)}{E_{p}}\left[1-\frac{\left(\Phi+2 \bar{\Phi} e^{-E^{(+)} / T}+e^{-2 E^{(+)} / T}\right) e^{-E^{(+)} / T}}{g^{(+)}(M, \Phi, \bar{\Phi} ; T, \mu ; p)}\right. \\
& \left.-\frac{\left(\bar{\Phi}+2 \Phi e^{-E^{(-)} / T}+e^{-2 E^{(-)} / T}\right) e^{-E^{(-)} / T}}{g^{(-)}(M, \Phi, \bar{\Phi} ; T, \mu ; p)}\right], \\
& b_{2}(T) \bar{\Phi}+b_{3} \Phi^{2}-b_{4}(\bar{\Phi} \Phi) \bar{\Phi}=-\frac{12 N_{f}}{T^{3}} \int \frac{d^{3} p}{(2 \pi)^{3}}\left[\frac{e^{-E^{(+)} / T}}{g^{(+)}(M, \Phi, \bar{\Phi} ; T, \mu ; p)}+\frac{e^{-2 E^{(-)} / T}}{g^{(-)}(M, \Phi, \bar{\Phi} ; T, \mu ; p)}\right], \\
& b_{2}(T) \Phi+b_{3} \bar{\Phi}^{2}-b_{4} \Phi(\bar{\Phi} \Phi)=-\frac{12 N_{f}}{T^{3}} \int \frac{d^{3} p}{(2 \pi)^{3}}\left[\frac{e^{-2 E^{(+)} / T}}{g^{(+)}(M, \Phi, \bar{\Phi} ; T, \mu ; p)}+\frac{e^{-E^{(-)} / T}}{g^{(-)}(M, \Phi, \bar{\Phi} ; T, \mu ; p)}\right] \text {. }
\end{aligned}
$$

As noted above, the role of $\Phi$ and $\bar{\Phi}$ are exchanged under charge conjugation $(\mu \rightarrow-\mu)$. This symmetry is reflected in the relation between the gap equations (2.19) and (2.20) under the charge conjugation transformation. In particular, this relation implies that $\Phi=\bar{\Phi}$ for $\mu=0$. Furthermore, we note that for $\Phi=\bar{\Phi}=1$, Eq. (2.18) is reduced to the gap equation of the standard NJL model without any coupling to the color $\mathrm{SU}_{c}(3)$ gauge field.

\section{THERMODYNAMIC QUANTITIES AT FINITE QUARK CHEMICAL POTENTIALS}

The thermodynamics of the PNJL model in the meanfield approximation is characterized by the potential $\Omega(\Phi, \bar{\Phi}, M)$ introduced in Eq. (2.15). In Figs. 1 and 2 we show the effective potential of the Polyakov loop $\mathcal{U}$ as well as the PNJL thermodynamic potential $\Omega$ in the chiral limit, at a high temperatures where chiral symmetry is restored and the dynamical quark mass $M$ vanishes. Consequently, only the Polyakov loop and its conjugate are the relevant classical fields in the problem.

For vanishing quark chemical potential, $\Phi$ and $\bar{\Phi}$ are equivalent. Hence, the thermodynamic potential is characterized by only one variable $\Phi$ that for a given temperature is determined by the gap equation (2.19). In Fig. 1 we show the $\Phi$ dependence of $\mathcal{U}$. This potential exhibits the expected structure in the $Z(3)$ symmetry broken phase with a minimum at a finite value of $\Phi$ below unity.

The influence of quarks on the PNJL model thermodynamics is illustrated in Fig. 2 where the $\Phi$-dependence of $\Omega$ is shown for the same temperature, $T=0.5 \mathrm{GeV}$. A comparison of Figs. 1 and 2 clearly shows that the interactions of the effective gluon field with the quarks leads to a shift of the minimum to larger values of $\Phi$. At high temperatures the minimum corresponds to $\Phi>1$.

The dynamical quark mass $M$ and the traced Polyakov loop $\Phi$ are the order parameters of the chiral and $Z(3)$ symmetries respectively. Thus, the $T$ and $\mu$ dependence of these order parameters is used to identify the phase boundaries of the model. In Figs. 3 and 4 we show $\Phi$ and

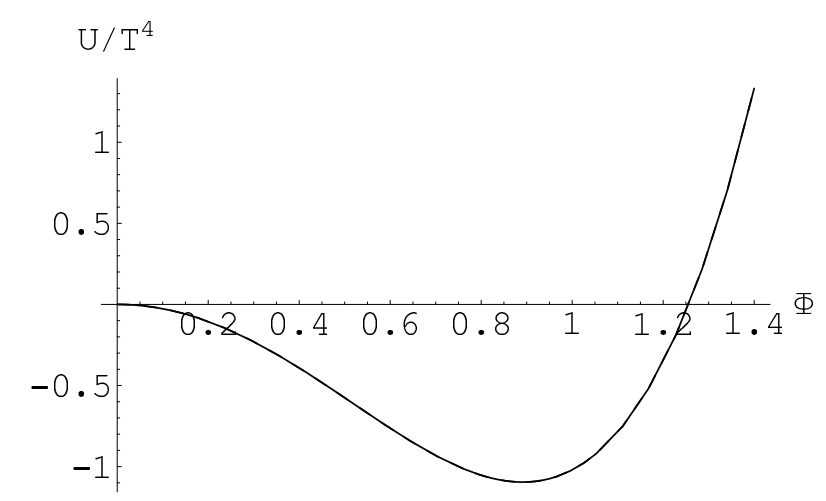

FIG. 1: The Polyakov loop effective potential $\mathcal{U} / T^{4}$ as a function of $\Phi$ at $T=500 \mathrm{MeV}$ in the chiral limit.

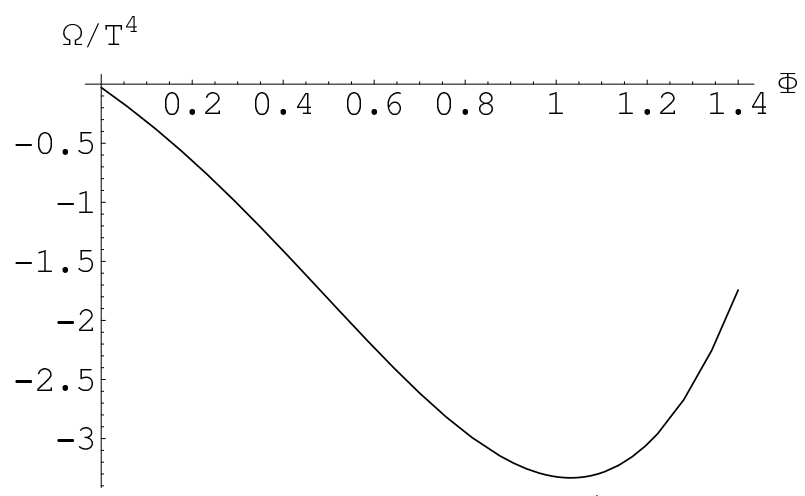

FIG. 2: The thermodynamic potential $\Omega / T^{4}$ as a function of $\Phi$ at $T=500 \mathrm{MeV}$ in the chiral limit.

$M$ at $\mu=0$, as functions of $T$ in the chiral limit. The phase change of the model is clearly indicated by rapid changes of the order parameters. The Polyakov loop potential $\mathcal{U}$ introduced in the PNJL Lagrangian preserves the invariance under the $Z(3)$ symmetry. However, due to the interactions with the quarks this symmetry is explicitly broken in the model. Thus, the Polyakov loop is not an order parameter in the strict sense and the transition is a rapid cross over. This is a remnant of the "deconfinement" phase transition of the pure Polyakov 


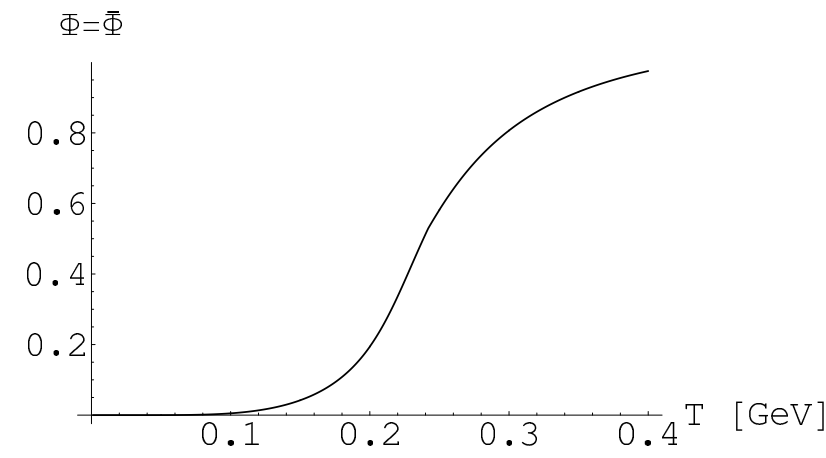

FIG. 3: Expectation value of the traced Polyakov loop $\Phi$ in the chiral limit as a function of temperature $T$ for vanishing quark chemical potential.

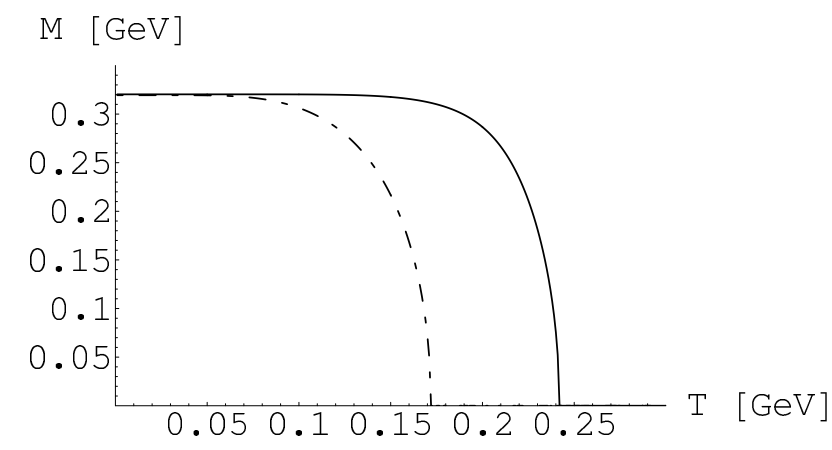

FIG. 4: The dynamical quark mass $M$ in the chiral limit as a function of temperature $T$ for a vanishing quark chemical potential. The dash-dotted line denotes the result obtained in the NJL model.

loop model. On the other hand, in the chiral limit the chiral transition is a true second order phase transition, with an order parameter $M$, which is strictly zero for temperatures above $T \simeq 242 \mathrm{MeV}$.

In Fig. 4 the dynamical quark mass $M$ obtained in the PNJL model is also compared with that of the NJL model \#3. It is clear that the coupling of the quarks to the effective gluon field shifts the chiral phase transition temperature to the higher value. This is a consequence of attractive interactions; in the presence of the Polyakov loop there is stronger "binding" of the constituent quarks in the chirally broken phase.

The non-locality of the quark interactions introduced by the form factor $F(x)$ also affects the critical temperature of the chiral phase transition. Suppressing the form factor in the PNJL Lagrangian results in a lower transition temperature. With the parameters used in our actual calculations, the reduction of $T_{c}$ is on the order of

\#3 In the NJL model, without Polyakov loops, we use the local version of the cutoff, i.e., a sharp cutoff. The cutoff is not implemented in the thermal part, which is anyway convergent.
$15 \mathrm{MeV}$, if in the thermal part of the PNJL model the momentum cutoff is not implemented.

The introduction of a finite quark chemical potential is expected to modify the behavior of the order parameters. The $\mu$ dependence of $M, \Phi$ and $\bar{\Phi}$ are shown in Figs. 5 and 6. There is a clear shift of the chiral transition to lower temperatures with increasing $\mu$ as is seen in Fig. 6. At a non-zero quark chemical potential the charge conjugation symmetry is broken, which leads to a splitting between $\Phi$ and $\bar{\Phi}$. As seen in Fig. 5 the Polyakov loop $\Phi$ is decreasing whereas $\bar{\Phi}$ is increasing with $\mu$. This is expected due to the relation of the Polyakov loop and its conjugate to the free energy of a quark and an antiquark respectively [20]. In a system with more quarks than antiquarks it is relatively easy to screen a static antiquark by a quark, forming a virtual $q \bar{q}$ state, whereas a static quark can only be screened by a diquark, thereby forming a colorless three-quark state.

At finite quark chemical potential, the order of the chiral phase transition can change. This is illustrated in Fig. 7 where the phase diagram of the PNJL model is shown. At low temperatures and large $\mu$ the transition is first order. The first order transition terminates at the tricritical point (TCP). With the actual value of the model parameters, summarized in Table. III, the TCP appears at $\left(T_{T C P}=157, \mu_{T C P}=266\right) \mathrm{MeV}$. Beyond the TCP, at smaller chemical potentials, the transition is second order. This is consistent with the gross structure of the phase diagram, expected for QCD according to universality arguments [21].

We explore the influence of the Polyakov loop on the thermodynamics, by considering observables that are related to the conservation of the net-quark number such as the quark number density $n_{q}(T, \mu)$ and the corresponding susceptibility $\chi_{q}(T, \mu)$. Both observables are obtained as derivatives of the thermodynamic potential $\Omega$ with respect to $\mu$. The quark number density is obtained from

$$
n_{q}=-\frac{\partial \Omega}{\partial \mu} .
$$

With the thermodynamic potential of the PNJL model (2.15) one finds

$$
\begin{aligned}
n_{q}= & 6 N_{f} \int \frac{d^{3} p}{(2 \pi)^{3}}\left[\frac{e^{-E^{(+)} / T}}{g^{(+)}}\right. \\
& \times\left(\Phi+2 \bar{\Phi} e^{-E^{(+)} / T}+e^{-2 E^{(+)} / T}\right) \\
& \left.-\frac{e^{-E^{(-)} / T}}{g^{(-)}}\left(\bar{\Phi}+2 \Phi e^{-E^{(-)} / T}+e^{-2 E^{(-)} / T}\right)\right] .
\end{aligned}
$$

The temperature dependence of the net-quark number density obtained with Eq. (3.2) is shown in Figs. 8 and 9 for various values of $\mu$. The PNJL model results are also compared with those of the NJL model. Clearly there is a substantial change in $n_{q}$ when the Polyakov loop dynamics is included. 

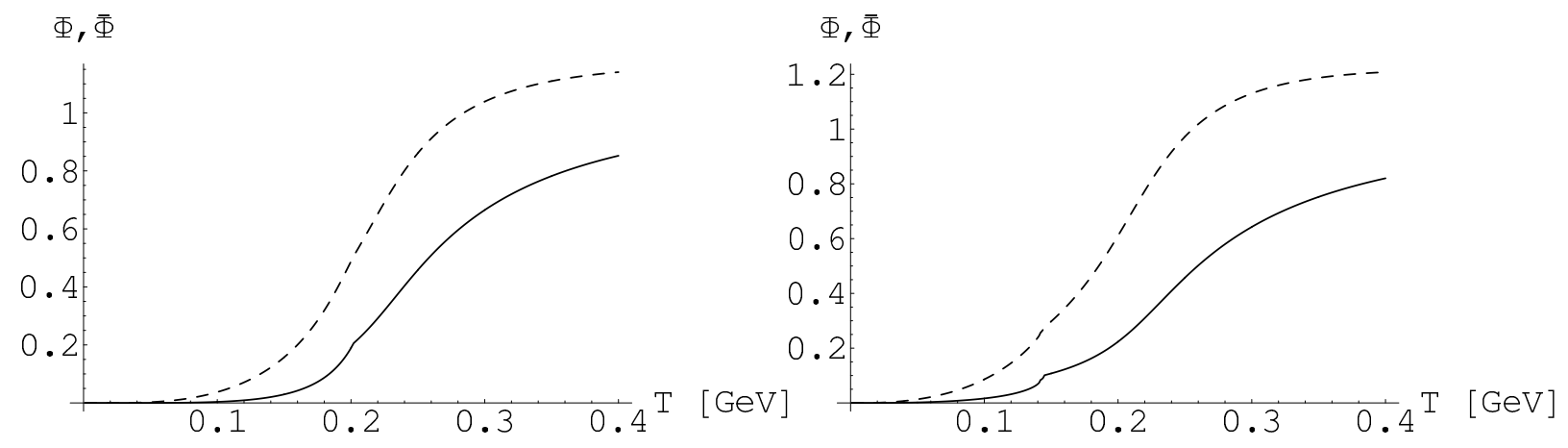

FIG. 5: Expectation values of the traced Polyakov loop $\Phi$ (solid) and $\bar{\Phi}$ (dashed) in the chiral limit as a function of temperature $T$ for finite quark chemical potentials. The lines in the left figure show $\Phi$ and $\bar{\Phi}$ at $\mu=200 \mathrm{MeV}$ and the chiral phase transition is of second order. The right figure is the results obtained at $\mu=270 \mathrm{MeV}$, which correspond to the first order chiral phase transition.

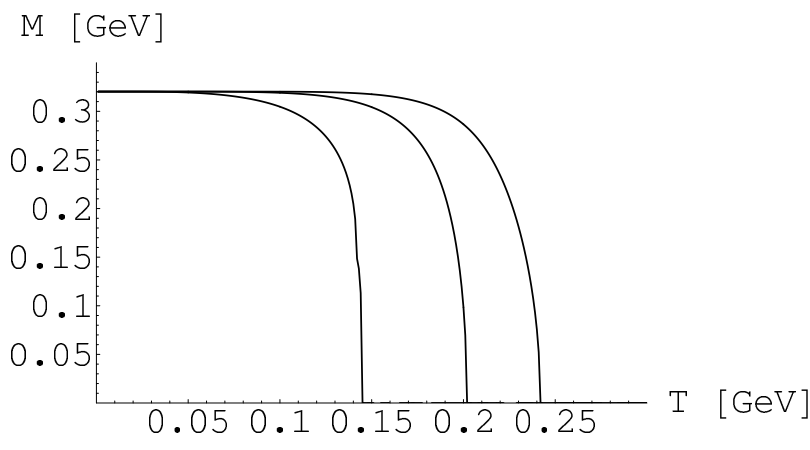

FIG. 6: The dynamical quark mass $M$ in the chiral limit as a function of temperature $T$ for finite quark chemical potentials $\mu=0,200 \mathrm{MeV}, 270 \mathrm{MeV}$ from right to left.

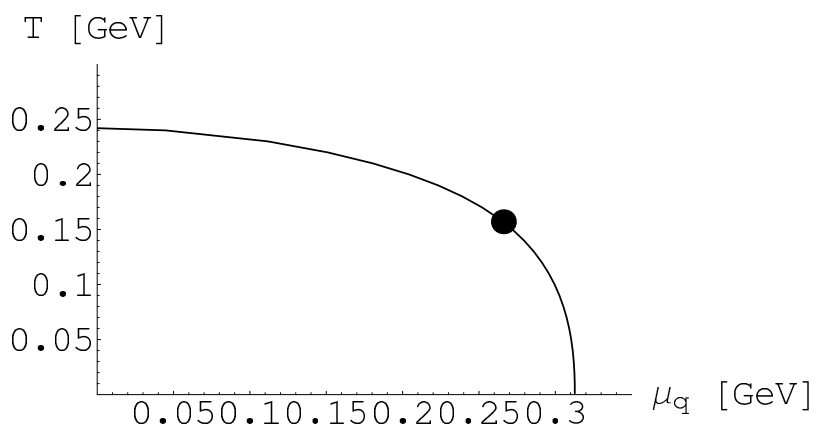

FIG. 7: The phase diagram of the PNJL model in the chiral limit. The dot indicates the location of the tricritical point, located at $\left(T_{T C P}=157, \mu_{T C P}=266\right) \mathrm{MeV}$.

In the region above the chiral phase transition the standard NJL model shows a relatively strong decrease of $n_{q} / T^{3}$ with increasing temperature. This behavior exactly reproduces the temperature dependence of a noninteracting gas of massless quarks, $n_{q}=N_{f}\left(\mu^{3} / \pi^{2}+\right.$ $\left.\mu T^{2}\right)$. Thus, the leading term in $n_{q} / T^{3}$ is proportional to $\mu / T$. Furthermore, the quark density increases as the

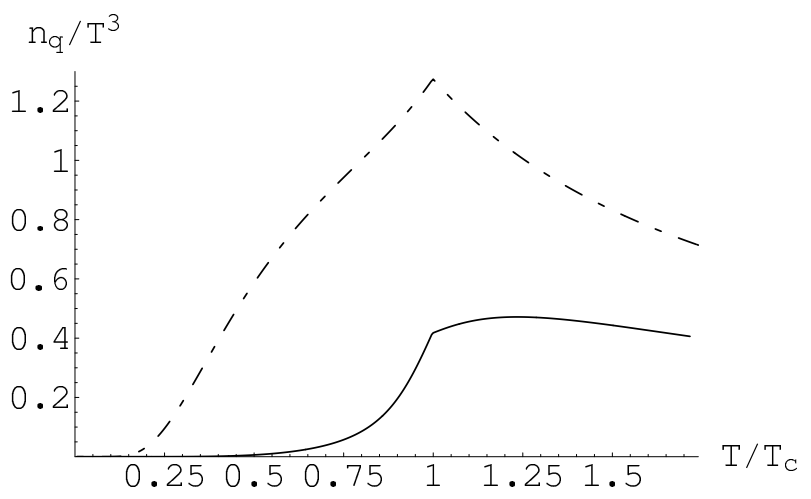

FIG. 8: The net-quark number density $n_{q} / T^{3}$ in the chiral limit as a function of temperature $T$ at $\mu=100 \mathrm{MeV}$. The dash-dotted line denotes the result obtained in the NJL model.

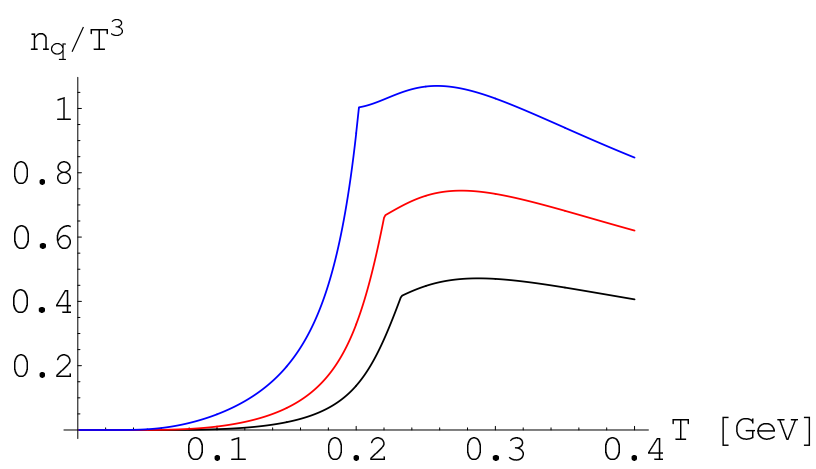

FIG. 9: The net-quark number density $n_{q} / T^{3}$ in the chiral limit as a function of temperature $T$. The lines, starting from the lowest one, correspond $\mu=100,150,200 \mathrm{MeV}$.

critical temperature $T_{c}$ is approached from below. This is due to the decrease of the effective quark mass as the chiral symmetry is restored. The PNJL model shows a substantially different temperature dependence of the 
quark density. First, there is a suppression of the oneand two-quark contributions to the density below $T_{c}$, due to the interactions with the effective gluon field. Hence, the leading contribution to the net quark density is due to the three-quark states. Clearly this leads to a strong suppression of the quark density below $T_{c}$. Above $T_{c}$, the suppression is much less effective. However, because $\Phi$ is still less than unity, a suppression compared to the quark density of a free gas at the same temperature and chemical potential remains.

The quark number susceptibility measures the response of the quark number density to changes in the quark chemical potential. This observable is of particular interest for exploring tricritical point. This is because $\chi_{q}$ is expected to diverge at TCP. This divergence is a remnant of the diverging fluctuations of the scalarisoscalar sigma field 22, 23, 24]. The net-quark number susceptibility is defined by

$$
\chi_{q}=\frac{\partial n_{q}}{\partial \mu} .
$$

In the PNJL model the dynamical quark mass $M$ and the Polyakov loops $\Phi, \bar{\Phi}$ implicitly depend on $\mu$. Thus, besides an explicit $\mu$-dependent contribution through Eq. (3.3) there are also terms proportional to $\mu$-derivatives of the effective condensates. In the PNJL model one finds

$$
\begin{aligned}
\chi_{q} & =\chi_{q}^{(0)}+T^{2} A_{M}^{(\mu)} \frac{\partial M}{\partial \mu}+T^{3} A_{\Phi}^{(\mu)} \frac{\partial \Phi}{\partial \mu}+T^{3} A_{\bar{\Phi}}^{(\mu)} \frac{\partial \bar{\Phi}}{\partial \mu} \\
& \equiv \chi_{q}^{(0)}+\chi_{q}^{(M)}+\chi_{q}^{(\Phi)}+\chi_{q}^{(\bar{\Phi})},
\end{aligned}
$$

where $\chi_{q}^{(0)}$ corresponds to the 0 -th order contribution given by

$$
\begin{aligned}
\chi_{q}^{(0)}= & \frac{6 N_{f}}{T} \int \frac{d^{3} p}{(2 \pi)^{3}}\left[\frac { e ^ { - E ^ { ( + ) } / T } } { ( g ^ { ( + ) } ) ^ { 2 } } \left\{\Phi+4 \bar{\Phi} e^{-E^{(+)} / T}\right.\right. \\
& +3(1+\bar{\Phi} \Phi) e^{-2 E^{(+)} / T}+4 \Phi e^{-3 E^{(+)} / T} \\
& \left.\left.+\bar{\Phi} e^{-4 E^{(+)} / T}\right\}+(\bar{\Phi}, \Phi ;-\mu)\right]
\end{aligned}
$$

The functions $A^{(\mu)}(M, \Phi, \bar{\Phi} ; T, \mu)$ and the $\mu$-derivatives of the condensates are introduced in Appendix B.

The quark number susceptibility at vanishing and at finite $\mu$ is shown as a function of the temperature in Figs. 10 and 11 for the PNJL and NJL models. At high temperature $\chi_{q}$ approaches the ideal gas limit, $\chi_{q} / T^{2} \simeq 2$. In the chiral limit, the quark number susceptibility has a discontinuity at the chiral phase transition at finite $\mu$, as found in Landau-Ginzburg theory 22] (see also [25, 26] for a detailed discussion of the quark number susceptibility in the NJL model).

The effect of quark-gluon interactions on the quark number susceptibility, shown in Fig. 10, is similar to that discussed in the context of the net-quark density. There is a reduction of the quark fluctuations below $T_{c}$ in the PNJL model relative to the fluctuations obtained

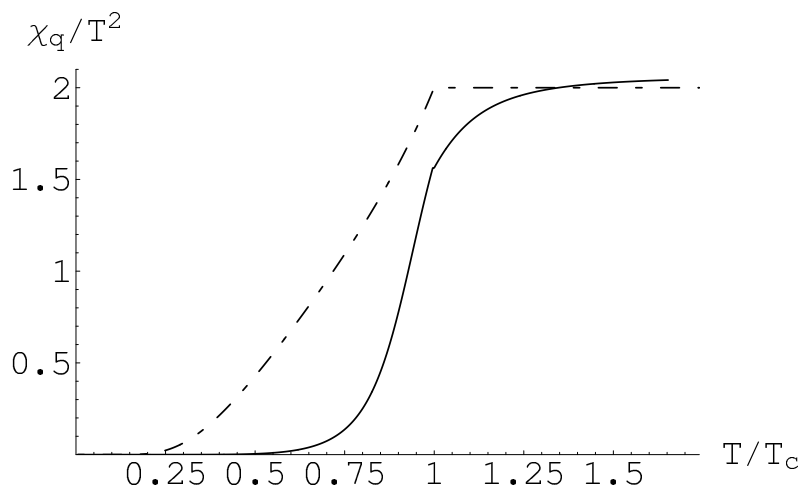

FIG. 10: The quark number susceptibility $\chi_{q} / T^{2}$ in the chiral limit as a function of temperature $T$, at $\mu=0$. The dashdotted line denotes the result obtained in the NJL model.

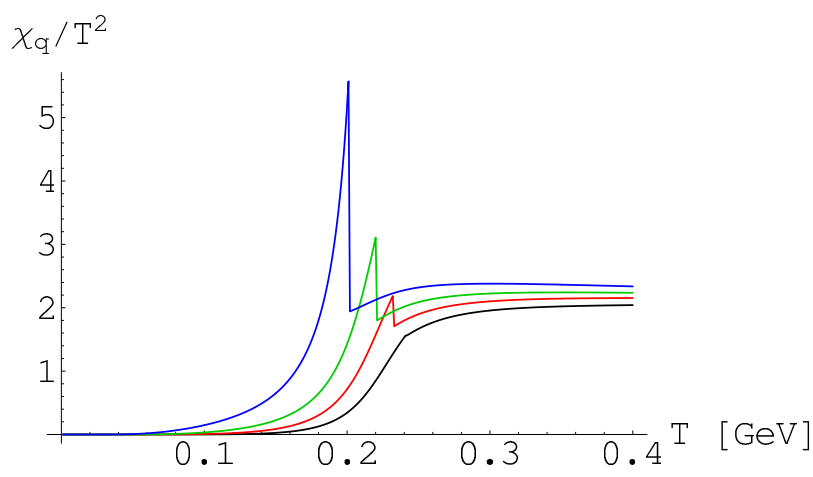

FIG. 11: The quark number susceptibility $\chi_{q} / T^{2}$ in the chiral limit as a function of temperature $T$. The lines correspond to the results in the PNJL model at $\mu=0,100,150,200 \mathrm{MeV}$ from below.

in NJL calculations. The PNJL results for the $T$ and $\mu$ dependence of $\chi_{q}$ and $n_{q}$ are in good agreement with recent LGT calculations of these quantities [27]. Thus, in contrast to the NJL model, the PNJL model provides a quantitative description of QCD thermodynamics near the phase transition.

At the TCP the quark number susceptibility diverges as shown in the left panel of Fig. 12 We now analyze the different contributions in Eq. (3.4). The right panel of Fig. 12 clearly shows that the singular behavior of $\chi_{q}$ is caused by the divergence of $\chi_{q}^{(M)}$. Both $\chi_{q}^{(\Phi)}$ and $\chi_{q}^{(\bar{\Phi})}$ have sharp peak structures at $T_{c}$, but, in contrast to $\chi_{q}^{(M)}$, they remain finite .

In order to understand the relation between the singular contributions to the susceptibilities, we consider an effective Lagrangian of the Landau type [28]

$\Omega(T, \mu) \simeq \Omega_{0}(T, \mu)+m M+\frac{1}{2} a(T, \mu) M^{2}+\frac{1}{4} b(T, \mu) M^{4}$,

where $a(T, \mu)$ and $b(T, \mu)$ are temperature dependent coefficients and $m$ is a source term, which breaks the sym- 


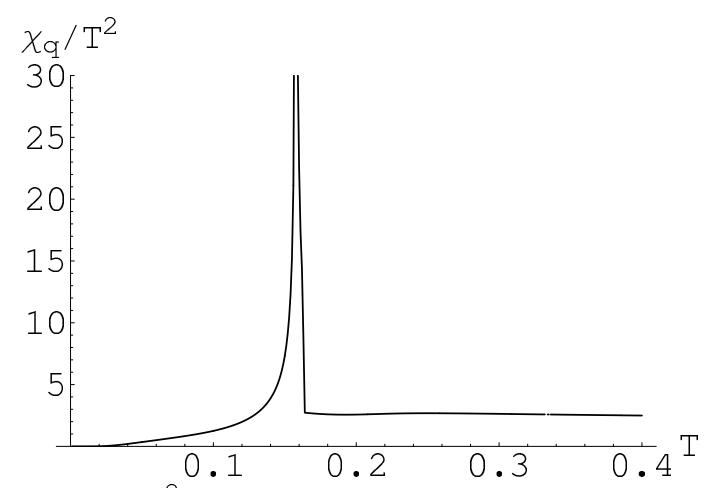

$[\mathrm{GeV}]$
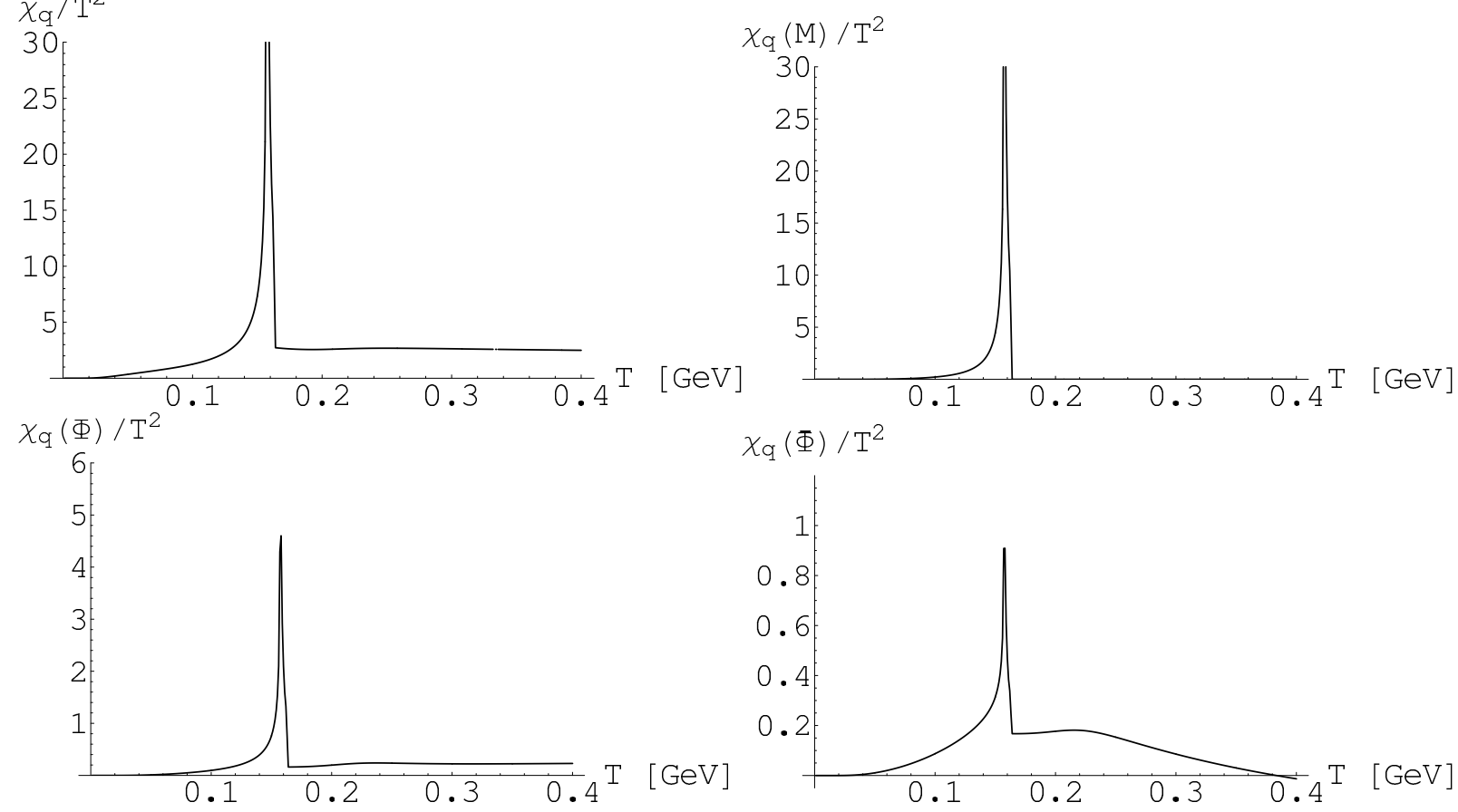

FIG. 12: The quark number susceptibility $\chi_{q} / T^{2}$ in the chiral limit as a function of temperature $T$ at the TCP $\mu=266 \mathrm{MeV}$. The components $\chi_{q}^{(M)}, \chi_{q}^{(\Phi)}$ and $\chi_{q}^{(\bar{\Phi})}$ as a function of $T$ at the TCP are defined in Eq. (3.4).

metry explicitly. At the end of the calculation we consider the "chiral limit", and set $m=0$. The equilibrium value of the order parameter $M$ is determined by the gap equation $\partial \Omega / \partial M=0$. In the broken symmetry phase $M=\sqrt{-a / b}$, while in the symmetric phase $M=0$. The model exhibits a second order transition at $a=0$ for $b>0$, and a tricritical point at $a=b=0$ [see e.g., Ref. [22]]. The chiral susceptibility

$$
\chi_{m m}=\frac{\partial M}{\partial m}=\frac{1}{a(T, \mu)+3 M^{2} b(T, \mu)},
$$

diverges along the second order transition line as well as at the TCP in the broken phase.

On the other hand, the singular part of the quark number susceptibility is given by

$$
\chi_{q}^{(M)}=-\frac{\partial a}{\partial \mu} M \frac{\partial M}{\partial \mu} .
$$

By using the gap equation one finds

$$
\frac{\partial M}{\partial \mu} \sim M \chi_{m m},
$$

which implies

$$
\chi_{q}^{(M)} \sim M^{2} \chi_{m m} \sim \frac{1}{b(T, \mu)},
$$

where in the final step we used the gap equation to eliminate $M$. Thus, the quark number susceptibility diverges only at the TCP. The mixing of the chiral and quark number susceptibilities can also be interpreted as a consequence of $\sigma-\omega$ mixing at finite baryon density [22, 23, 24, 26].

\section{CHIRAL AND POLYAKOV LOOP SUSCEPTIBILITIES}

In the PNJL model the constituent quarks and the Polyakov loops are effective fields related with the order parameters for the chiral and $Z(3)$ symmetry breaking. In LGT the susceptibilities associated with these fields show clear signals of the phase transitions. In this section we present the calculational scheme for computing the susceptibilities and discuss their relation to the spontaneous breaking of the chiral and $Z(3)$ symmetries.

Consider first the generating functional of a scalar field theory

$$
Z[J]=e^{-i W[J]}=\int \mathcal{D} \phi \exp \left[i \int d^{4} x(\mathcal{L}[\phi]+J \phi)\right],
$$

where $J$ is an external source. The second functional derivative of $W[J]$ with respect to $J$ yields the correlation function:

$$
\left.\frac{\delta^{2} W[J]}{\delta J(x) \delta J(y)}\right|_{J=0}=\langle\phi(x) \phi(y)\rangle-\langle\phi(x)\rangle\langle\phi(y)\rangle .
$$

The effective action $\Gamma[\phi]$ is introduced through the Leg- 
endre transformation

$$
\Gamma[\phi]=-W[J]-\int d^{4} x J(x) \phi(x) .
$$

The correlation function introduced in the Eq. (4.2) can now be expressed by means of

$$
\left.\frac{\delta^{2} W[J]}{\delta J(x) \delta J(y)}\right|_{J=0}=\left(\frac{\delta^{2} \Gamma[\phi]}{\delta \phi(x) \delta \phi(y)}\right)^{-1} .
$$

Thus, by using this identity, one can compute the susceptibility also from the effective potential, by taking derivatives with respect to the classical field $\phi$.

In the case of the PNJL model there are three different classical fields $\vec{\phi}=(M, \Phi, \bar{\Phi})$ that correspond to the order parameters; the dynamical quark mass, the Polyakov loop and its complex conjugate. Consequently, in order to compute the corresponding susceptibilities from the effective potential, the relation (4.4) must be generalized. We use the chain rule,

$$
\delta_{i j}=\frac{\delta \phi_{i}}{\delta \phi_{j}}=\frac{\delta}{\delta \phi_{j}} \frac{\delta W}{\delta J_{i}}=\frac{\delta J_{k}}{\delta \phi_{j}} \frac{\delta^{2} W}{\delta J_{k} \delta J_{i}}=\frac{\delta^{2} \Gamma}{\delta \phi_{j} \delta \phi_{k}} \frac{\delta^{2} W}{\delta J_{k} \delta J_{i}},
$$

where $\vec{J}=\left(J_{M}, J_{\Phi}, J_{\bar{\Phi}}\right)$ is a vector composed of the source fields corresponding to the order parameters in $\vec{\phi}$.

It follows from Eq. (4.5) that the susceptibilities are obtained by inverting the matrix composed of the second derivatives of the effective action with respect to classical fields. Following Ref. [3], we introduce a dimensionless matrix

$$
\hat{C}=\left(\begin{array}{ccc}
C_{m m} & C_{m l} & C_{m \bar{l}} \\
C_{l m} & C_{l l} & C_{l \bar{l}} \\
C_{\overline{l m}} & C_{\overline{l l}} & C_{\overline{l l}}
\end{array}\right),
$$

with the components

$$
\begin{aligned}
& C_{m m}=\frac{1}{T \Lambda} \frac{\partial^{2} \Omega}{\partial M^{2}}, \quad C_{l l}=\frac{1}{T \Lambda^{3}} \frac{\partial^{2} \Omega}{\partial \Phi^{2}}, \\
& C_{\overline{l l}}=\frac{1}{T \Lambda^{3}} \frac{\partial^{2} \Omega}{\partial \bar{\Phi}^{2}}, \quad C_{l \bar{l}}=C_{\overline{l l}}=\frac{1}{T \Lambda^{3}} \frac{\partial^{2} \Omega}{\partial \Phi \partial \bar{\Phi}}, \\
& C_{m l}=C_{l m}=\frac{1}{T \Lambda^{2}} \frac{\partial^{2} \Omega}{\partial M \partial \Phi} \\
& C_{m \bar{l}}=C_{\overline{l m}}=\frac{1}{T \Lambda^{2}} \frac{\partial^{2} \Omega}{\partial M \partial \bar{\Phi}} .
\end{aligned}
$$

Through Eq. (4.5) a set of susceptibilities is defined by

$$
\hat{\chi}=\left(\begin{array}{ccc}
\chi_{m m} & \chi_{m l} & \chi_{m \bar{l}} \\
\chi_{l m} & \chi_{l l} & \chi_{l \bar{l}} \\
\chi_{\overline{l m}} & \chi_{\overline{l l}} & \chi_{\overline{l l}}
\end{array}\right),
$$

where $\chi_{i j}$ is given by the inverse of $\hat{C}$,

$$
\chi_{i j}=\left(\hat{C}^{-1}\right)_{i j} \quad i, j=\{m, l, \bar{l}\} .
$$

Here $\chi_{m m}$ is the chiral susceptibility, as in the previous section, while $\chi_{l l}$ and $\chi_{\overline{l l}}$ are the diagonal Polyakov loop susceptibilities (see below). The off-diagonal terms correspond to mixed susceptibilities.

In the pure gluon sector, the susceptibilities are related to the fluctuations of the $\Phi$ and $\bar{\Phi}$ fields. Under the $Z(3)$ transformation $\bar{\Phi} \Phi, \Phi^{3}, \bar{\Phi}^{3}$ and combinations thereof are invariant. Thus, the off-diagonal susceptibility $\chi_{l \bar{l}}$ is $Z(3)$ invariant, while the diagonal pieces $\chi_{l l, \overline{l l}}$ are not:

$$
\begin{aligned}
& \chi_{l \bar{l}}=\langle\bar{\Phi} \Phi\rangle-\langle\bar{\Phi}\rangle\langle\Phi\rangle, \\
& \chi_{l l}=\left\langle\Phi^{2}\right\rangle-\langle\Phi\rangle^{2}, \\
& \chi_{\overline{l l}}=\left\langle\bar{\Phi}^{2}\right\rangle-\langle\bar{\Phi}\rangle^{2} .
\end{aligned}
$$

For vanishing quark chemical potential, $\chi_{l l}$ and $\chi_{\overline{l l}}$ coincide but are not equal to $\chi_{l \bar{l}}$. In the low-temperature phase, where the $Z(3)$ symmetry is realized, the $Z(3)$ non-invariant components $\chi_{l l}$ and $\chi_{\overline{l l}}$ are strongly suppressed ${ }^{\# 4}$. In the $Z(3)$ symmetry broken phase at high temperatures, all components of the Polyakov loop susceptibility are large.

We also define the average susceptibility

$$
\bar{\chi}_{l l}=\frac{1}{4}\left(\chi_{l l}+\chi_{\overline{l l}}+2 \chi_{l \bar{l}}\right) .
$$

which corresponds to fluctuations of the real part of the Polyakov loop. This observable has been used in LGT calculations to identify the position of deconfinement transition in the QCD medium.

Due to the presence of dynamical quarks in the PNJL model, the $Z(3)$ symmetry is explicitly broken and the Polyakov loop is not a genuine order parameter. Nevertheless, the fact that in LGT calculations the expectation values of $\Phi$ and $\bar{\Phi}$ are strongly suppressed in the low temperature phase [29], suggests that the $Z(3)$ symmetry is a useful guiding principle for constructing model Lagrangians (see also Figs. 3 and 5 ).

\section{SUSCEPTIBILITIES AND THE PHASE TRANSITION IN THE PNJL MODEL}

Enhanced fluctuations are characteristic for phase transitions. Thus, the exploration of fluctuations is a promising tool for probing the phase structure of a system. The phase boundaries can be identified by the response of the fluctuations to changes in the thermodynamic parameters. In this section we focus on the phase structure of the PNJL model by studying the order parameter susceptibilities, defined in the previous section.

\footnotetext{
\#4 In purely gluonic theory, where the $Z(3)$ symmetry is exact, $\chi_{l l}$ and $\chi_{\overline{l l}}$ vanish identically in the low temperature phase.
} 


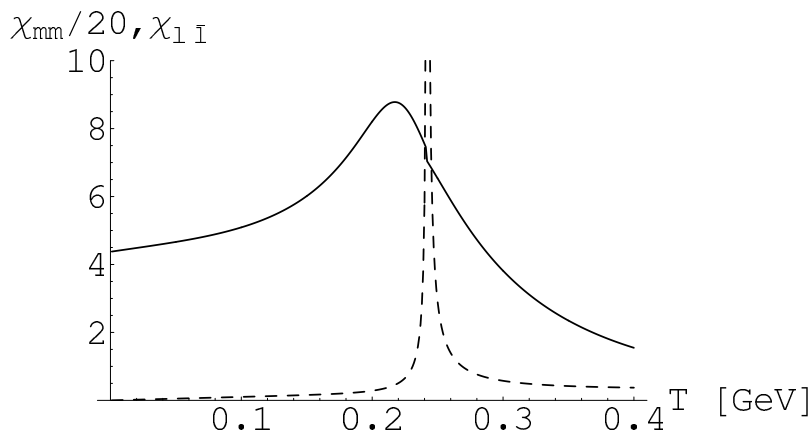

FIG. 13: The chiral $\chi_{m m}$ (dashed-line) and the Polyakov loop $\chi_{l \bar{l}}$ (solid-line) susceptibilities in the chiral limit as functions of temperature $T$ for $\mu=0$.

\subsection{Susceptibilities at vanishing quark chemical potential}

In Fig. 13 we show the chiral and Polyakov loop susceptibilities $\chi_{m m}$ and $\chi_{l \bar{l}}$ computed at $\mu=0$ in the PNJL model in the chiral limit. The chiral susceptibility exhibits a very narrow divergent peak at the chiral critical temperature $T_{\mathrm{ch}}$, while the Polyakov loop susceptibility shows a very different behavior: the peak is much broader and the susceptibility remains finite for all temperatures. The latter is due to the explicit breaking of the $Z(3)$ symmetry by the presence of the quark fields in the PNJL Lagrangian. Nevertheless, $\chi_{l \bar{l}}$ still exhibits a peak structure that can be considered as a signal for the deconfinement transition in this model.\#5 The peak position of $\chi_{l \bar{l}}$ appears at $T \simeq 217 \mathrm{MeV}$ below the chiral critical temperature $T_{\text {ch }} \simeq 242 \mathrm{MeV}$. Thus, in this model the deconfinement phase transition occurs at a lower temperature than that of the chiral phase transition. The separation between the two peaks is roughly $25 \mathrm{MeV}$ in the non-local model and about $8 \mathrm{MeV}$ in the local model.

Another interesting feature of $\chi_{l \bar{l}}$ is the interference with the chiral susceptibility seen in Fig. 13, At the chiral transition, $T=T_{\mathrm{ch}}$, there is a narrow structure in $\chi_{l \bar{l}}$. We stress that this feature is not related with the deconfinement transition, but only due to the coupling to the chiral susceptibility. Thus, for the parameters used in the model, the deconfinement transition, signaled by the broad bump in $\chi_{l \bar{l}}$, sets in earlier than the chiral transition at vanishing net quark density.

\footnotetext{
\#5 As noted above, a similar procedure is usually applied in LGT
} studies of QCD thermodynamics.

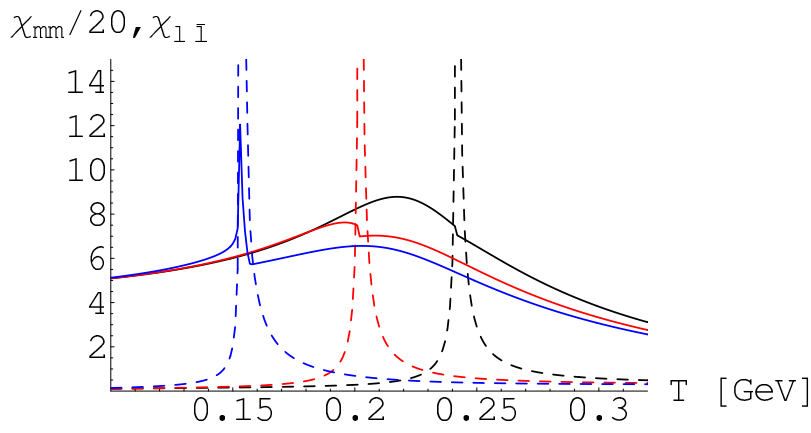

FIG. 14: The chiral $\chi_{m m}$ (dashed) and Polyakov loop $\chi_{l \vec{l}}$ (solid) susceptibilities in the chiral limit as functions of temperature $T$. The lines correspond to $\mu=0$ (right), $\mu=200$ $\mathrm{MeV}$ (middle) and $\mu=270 \mathrm{MeV}$ (left).

\subsection{Susceptibilities at a finite quark chemical potential}

At finite chemical potential, there is a shift of the chiral phase transition to lower temperatures, as shown in Fig. 7 This is consistent with recent LGT results at finite quark chemical potential [30]. A lowering of the deconfinement temperature is also expected at non-zero net quark density. The position of deconfinement and chiral transitions can be determined by exploring the order parameter susceptibilities introduced in the previous section. With increasing chemical potential the temperature dependence of the Polyakov loop expectation value is flattening and for sufficiently large $\mu_{q}$ it shows almost no variation with $T$. Consequently, the width of the Polyakov loop susceptibility is increasing with increasing $\mu_{q}$.

In Figs. 14 and 15 we illustrate the temperature dependence of the susceptibilities for several values of the chemical potential. With increasing $\mu$ the peak position of the chiral and Polyakov loop susceptibilities are clearly shifted towards lower $T$ and approach each other as seen in Fig. 14. At $\mu_{0} \simeq 185 \mathrm{MeV}$ the two peaks coincide, which indicates that the chiral and deconfinement transitions appear at the same temperature. For $\mu>\mu_{0}$ the Polyakov loop susceptibility $\chi_{\bar{l}}$ has a sharp peak at $T_{\mathrm{ch}}$ and a broad bump above $T_{\mathrm{ch}}$. A similar behavior was also found in Ref. [3]. The peak at $T_{\mathrm{ch}}$ in $\chi_{l \bar{l}}$ is clearly due to an interference with the chiral phase transition, while the bump corresponds to the pseudo-critical point of the deconfinement transition. This structure is also seen in $\chi_{l \bar{l}}$ at the tricritical point, located at $\left(T_{c}=157, \mu_{c}=266\right)$ $\mathrm{MeV}$.

In the previous section we also introduced the average susceptibility $\bar{\chi}_{l l}$, which corresponds to fluctuations of the real part of the Polyakov loop. In Fig. 15 the temperature variation of $\bar{\chi}_{l l}$ for different values of the quark chemical potential is shown. It is clear from this figure that a behavior of $\bar{\chi}_{l l}$ is very similar to that of $\chi_{l \bar{l}}$. However, the peak positions of the two susceptibilities differ 


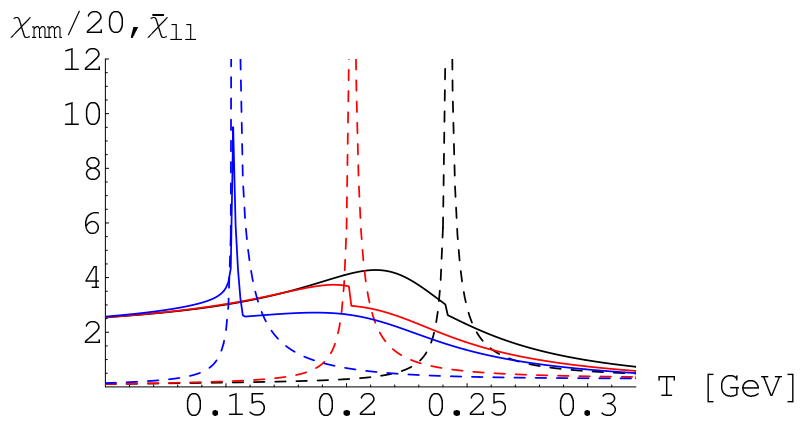

FIG. 15: The chiral $\chi_{m m}$ (dashed) and Polyakov loop $\bar{\chi}_{l l}$ (solid) susceptibilities in the chiral limit as functions of temperature $T$. The lines correspond to $\mu=0$ (right), $\mu=200$ $\mathrm{MeV}$ (middle) and $\mu=270 \mathrm{MeV}$ (left).

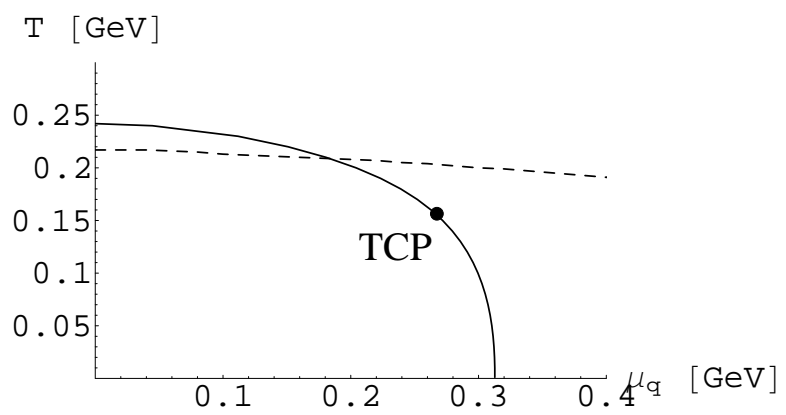

FIG. 16: The phase diagram of the PNJL model in the chiral limit. The solid (dashed) line denotes the chiral (deconfinement) phase transition respectively. The TCP (bold-point) is located at $\left(T_{c}=157, \mu_{c}=266\right) \mathrm{MeV}$. The parameter set (a) in Table III was used in the calculation.

slightly. Thus, in the Polyakov loop sector the determination of the transition temperature by using the susceptibilities is not unique. Nevertheless, the differences are small, so in the following we use $\chi_{l \bar{l}}$ to identify the deconfinement transition temperature in PNJL model.

The peak positions of the $\chi_{m m}$ and $\chi_{l \bar{l}}$ susceptibilities are used to determine the phase boundaries in the $(T, \mu)-$ plane. We stress that the phase boundaries are strongly dependent on the model parameters. In Fig. [16] we show the phase diagram for the PNJL model obtained with the parameters from Table I. Clearly the boundary line related with the chiral symmetry restoration, determined by the peak in the chiral susceptibility, coincides with that in Fig. 7 which was computed from the properties of the dynamical quark mass. Furthermore, the phase boundary corresponding to the deconfinement transition is identified with the position of the broad maximum observed in $\chi_{l \bar{l}}$. With these parameters the boundary lines of deconfinement and chiral symmetry restoration do not coincide. As anticipated in the discussion of Fig. 14 there is only one common point in the phase diagram where the

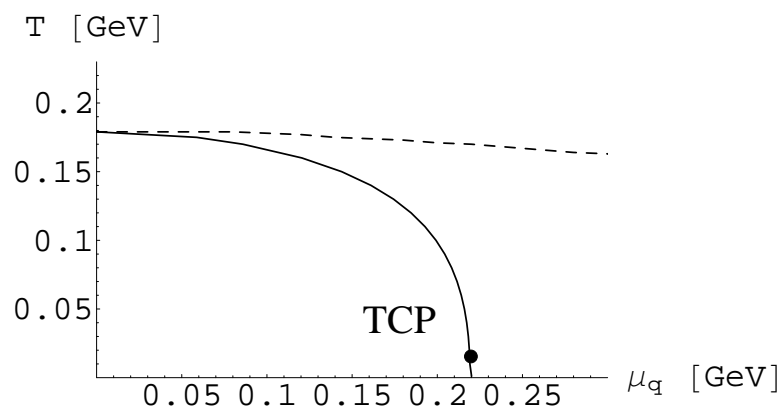

FIG. 17: The phase diagram of the PNJL model in the chiral limit. The solid (dashed) line denotes the chiral (deconfinement) phase transition respectively. The TCP (bold-point) is located at $\left(T_{c}=15, \mu_{c}=218\right) \mathrm{MeV}$. The results correspond to parameter set (b) in Table III

two transitions appear simultaneously.

Recent LGT results both at vanishing and at finite quark chemical potential show that deconfinement and chiral symmetry restoration appears in QCD along the similar critical line [31]. In general it is possible to choose the PNJL model parameters such that the critical temperatures of chiral and deconfinement transition coincide at $\mu=0$. The resulting phase diagram is shown in Fig. 17 the parameters used in the calculations are summarized in Table III set (b). We note that this choice of the parameters is not unique. A shift in the position of the critical temperature at $\mu=0$ can also be obtained by changing the $T_{0}$-parameter in the effective gluon potential. Decreasing $T_{0}$ from $270 \mathrm{MeV}$ to $130 \mathrm{MeV}$ results in $T_{\mathrm{ch}}=192 \mathrm{MeV}$, consistent with recent LGT calculations 32.

From Figs. 16 and 17 it is clear that in our model there is only a rather narrow region of finite $\mu$ where the deconfinement and chiral transition lines coincide. The slope of $T_{\mathrm{dec}}$ as a function of $\mu$ is almost flat, indicating that at low temperature the chiral phase transition should appear much earlier than deconfinement. So far there is no guidance available from first principle LGT calculations concerning the relation between deconfinement and chiral symmetry restoration at large values of the chemical potential. However, there are general arguments, that the deconfinement transition should precede restoration of chiral symmetry (see e.g. 33, 34]). In view of this, it seems unlikely that at $T \simeq 0$ the chiral symmetry sets in at the lower baryon density than deconfinement. In the PNJL model, the effective gluon potential parameters were fixed by fitting quenched LGT calculations. Consequently, the parameters are taken as independent on $\mu$. However, it is conceivable that the effect of dynamical quarks can modify the coefficients of this potential, thus resulting in $\mu$-dependence of the parameters. Consequently, the slope of $T_{\mathrm{dec}}$ as a function of $\mu$ could be 


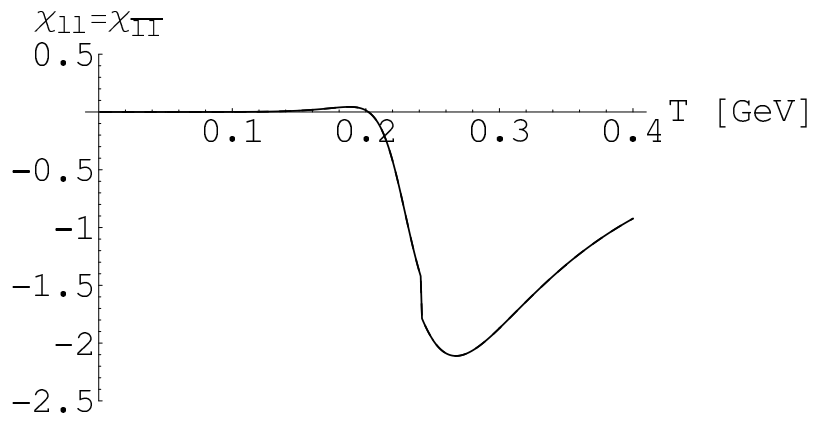

FIG. 18: The diagonal $\chi_{l l}=\chi_{\overline{l l}}$ susceptibility in the chiral limit as a function of temperature $T$ for $\mu=0$.

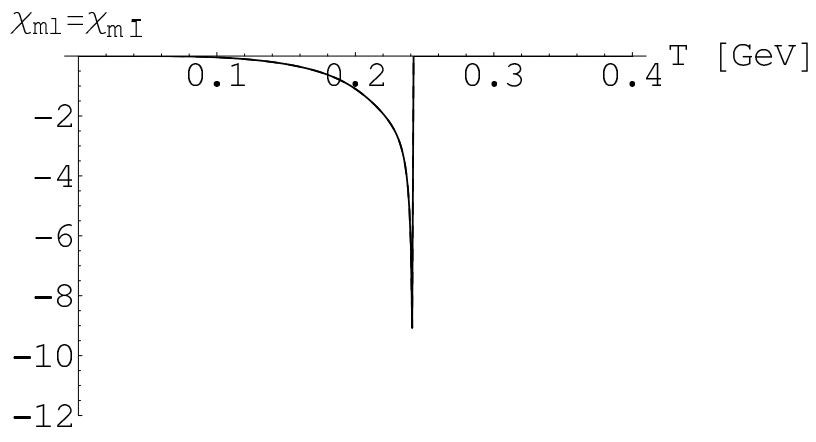

FIG. 19: The off-diagonal $\chi_{m l}=\chi_{m \bar{l}}$ susceptibility in the chiral limit as a function of temperature $T$ for $\mu=0$.

steeper ${ }^{\# 6}$. Consequently, the effective Polyakov loop potential (2.8) should, with $\mu$-independent coefficients, be employed only for $\mu / T<1$.

In Figs. 18 and 19 we show further diagonal and off-diagonal components of the susceptibility $\hat{\chi}(4.8)$ at $\mu=0$. In this case the diagonal components $\chi_{l l}$ and $\chi_{\overline{l l}}$ coincide. These are the $Z(3)$ non-invariant susceptibilities for $\Phi$ and $\bar{\Phi}$. These components are suppressed in the low-temperature phase, where the $Z(3)$ symmetry is (approximately) restored. Around $T \simeq T_{\mathrm{dec}}$ the $\chi_{l l, \overline{l l}}$ show a rapid drop, associated with the crossover transition, where the expectation values $\Phi$ and $\bar{\Phi}$ grow rapidly. In the high-temperature phase $\chi_{l l, \overline{l l}}$ are not necessarily suppressed since the $Z(3)$ symmetry is explicitly broken there. The off-diagonal susceptibilities $\chi_{m l}=\chi_{m \bar{l}}$ are also suppressed in the low-temperature phase and show a clear peak at $T_{\mathrm{ch}}$ due to an interference with the chiral phase transition. These susceptibilities are non-invariant under both the $Z(3)$ and chiral symmetries. Hence, they are suppressed well below the transition, where the $Z(3)$ symmetry is restored and vanish above $T_{\mathrm{ch}}$, in the chi-

\#6 Such a modification was explored in Ref. [35] where explicit $\mu$ and $N_{f}$ - dependence of $T_{0}$ is extracted from the running coupling constant $\alpha_{s}$, using the argument based on the renormalization group. rally symmetric phase.

An alternative way to determine the deconfinement and chiral transition temperatures, by locating the maximum of the temperature derivative of the corresponding effective condensate, has been discussed in the literature (see e.g. ref. [13]). The temperature derivatives of the condensates can be expressed as combinations of the susceptibilities with some $T$ - and $\mu$-dependent coefficients, as shown in Eq. (B.3). Consequently, in general the deconfinement pseudo critical temperature obtained with this method does not agree with that obtained from the peak position of the corresponding susceptibilities. Only if the phase transitions are relatively sharp and the susceptibilities show narrow structures can one expect that the transition temperatures determined using the different prescriptions coincide. In Fig. 20 we show the derivatives of order the parameters at $\mu=0$ for different values of $T_{0}$.

In ref. [5] the peak positions in the derivatives of the Polyakov loop and of the chiral order parameter coincide. Indeed, in the local version of the PNJL model with $T_{0}=270 \mathrm{MeV}$ we reproduce this result, as illustrated in Fig. 20. However, for a slightly different value of $T_{0}$, the positions of the two peaks split. The parameters in the Polyakov loop sector were fixed from the lattice data in the heavy quark mass limit. This corresponds to a transition temperature $T_{0}=270 \mathrm{MeV}$ in the pure gauge theory. In the presence of dynamical quarks the transition temperature drops, thus it is not excluded that the value of $T_{0}$ may depend on $N_{f}$, as noted in the introduction. With $T_{0}=210 \mathrm{MeV}$, the local PNJL model yields a splitting of the peaks in the derivatives of the Polyakov loop and the chiral condensate. Thus, the locations of the pseudo-critical points are strongly dependent on the model parameters. In the non-local PNJL model considered in this work, there is no coincidence between the peak positions and the peak of $\frac{\partial \Phi}{\partial T}$ is located at a lower temperature than that of $\frac{\partial M}{\partial T}$ for all values of $T_{0} \lesssim 300$ $\mathrm{MeV}$, as illustrated in Fig. 20 and c. We note that in the non-local PNJL model the chiral transition temperatures obtained, on the one hand, from the derivative of the order parameter and, on the other hand, from the corresponding susceptibility are almost identical. Thus, at least in the chiral limit, the two methods for identifying the chiral transition, are equivalent. However, for the Polyakov loop, the difference is $\sim 10 \mathrm{MeV}$ at $\mu=0$.

\subsection{Effective potential constraints}

As shown in Fig. 18, $\chi_{l l}$ is negative in a broad temperature range above $T_{\mathrm{ch}}$. This is in disagreement with recent lattice results, where $\chi_{l l}$ is always positive in the presence of dynamical quarks [31]. Furthermore, the relation of the Polyakov loop susceptibility with the free energy of static quarks is also incompatible with negative values of $\chi_{l l}$. A possible origin of this behavior could be the approximation to the effective Polyakov loop potential used 


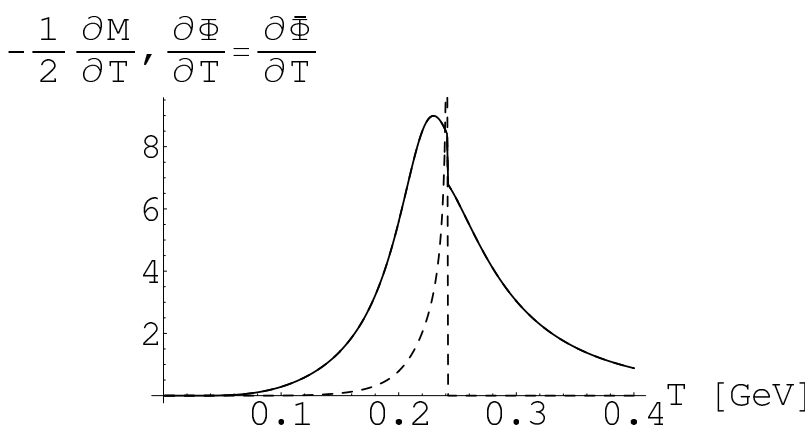

(a) $T_{0}=270 \mathrm{MeV}$, non-local

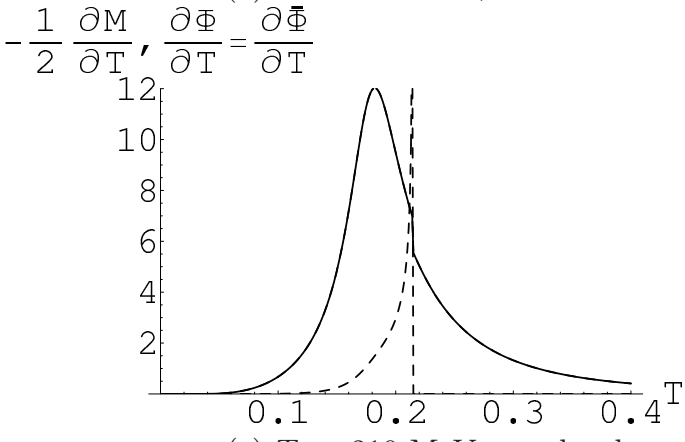

(c) $T_{0}=210 \mathrm{MeV}$, non-local $-\frac{1}{2} \frac{\partial M}{\partial T}, \frac{\partial \Phi}{\partial T}=\frac{\partial \Phi}{\partial T}$

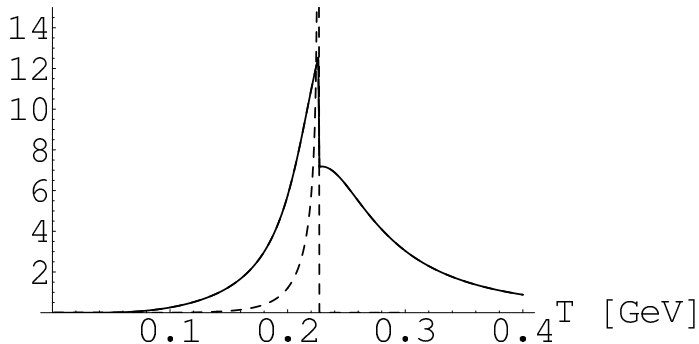

(b) $T_{0}=270 \mathrm{MeV}$, local

$-\frac{1}{2} \frac{\partial \mathrm{M}}{\partial \mathrm{T}}, \frac{\partial \Phi}{\partial \mathrm{T}}=\frac{\partial \Phi}{\partial \mathrm{T}}$

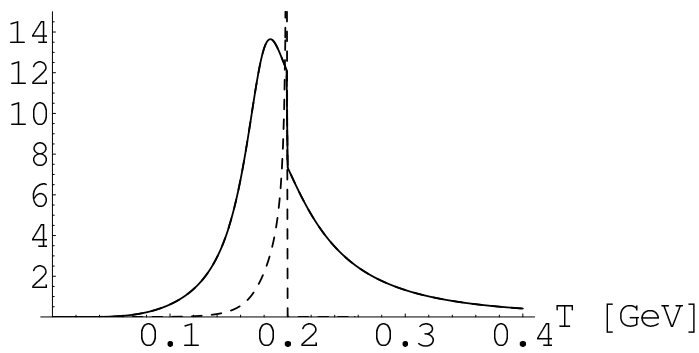

(d) $T_{0}=210 \mathrm{MeV}$, local

FIG. 20: Derivatives of the dynamical quark mass $\partial M / \partial T$ (dashed) and the Polyakov loop $\partial \Phi / \partial T$ (solid) in the chiral limit as functions of temperature $T$ for $\mu=0$.

in the Eq. (2.8).

The $\chi_{l l}$ susceptibility can be related to the screening masses $m_{r}$ and $m_{i}$ of the real and imaginary part of the Polyakov loop correlation functions [36]:

$$
\chi_{l l}=\frac{1}{m_{r}^{2}}-\frac{1}{m_{i}^{2}} .
$$

In Ref. 36] the ratio

$$
\frac{m_{i}}{m_{r}} \simeq 3
$$

was found in the vicinity to the critical temperature. This result is qualitatively consistent with the perturbative calculation [37]. From the Eqs. (5.1) and (5.2) it is clear that the resulting $\chi_{l l}$ should be positive at $T=T_{c}$.

In Ref. [36] the screening masses $m_{r}$ and $m_{i}$ were calculated with an effective potential that has the same polynomial form as used in the Eq. 2.8 but with different values of the parameters. With the values given in table II effective Polyakov loop potential (2.8) yields

$$
m_{i}<m_{r}
$$

which implies a negative value of $\chi_{l l}$ at $T_{c}$.

From this discussion it is clear that the behavior of $\chi_{l l}$ depends crucially on the parameters used in the Polyakov loop potential. Thus, the constraints from $Z(3)$ symmetry and from the lattice results for the equation of state, are not sufficient to warrant physically acceptable susceptibilities.
Recently an improved effective potential with temperature- dependent coefficients has been suggested 13 .

$$
\begin{aligned}
& \frac{\mathcal{U}(\Phi, \bar{\Phi} ; T)}{T^{4}}=-\frac{a(T)}{2} \bar{\Phi} \Phi \\
& +b(T) \ln \left[1-6 \bar{\Phi} \Phi+4\left(\Phi^{3}+\bar{\Phi}^{3}\right)-3(\bar{\Phi} \Phi)^{2}\right]
\end{aligned}
$$

where

$a(T)=a_{0}+a_{1}\left(\frac{T_{0}}{T}\right)+a_{2}\left(\frac{T_{0}}{T}\right)^{2}, \quad b(T)=b_{3}\left(\frac{T_{0}}{T}\right)^{3}$,

The polynomial in $\Phi$ and $\bar{\Phi}$, used in (2.8), is replaced by a logarithmic term, which accounts for the Haar measure in the group integral [3]. The parameters in (5.4) were fixed to reproduce the lattice results for pure gauge QCD thermodynamics and for the behavior of the Polyakov loop. These parameters are summarized in the Table IV.

In Fig. 21 we show the $\chi_{l l}$ susceptibility calculated with the potential of Eq. (5.4). It is clear from this figure that the improved potential yields positive values for the Polyakov loop susceptibilities . In addition the peak positions of the $\chi_{l l}$ and $\chi_{l \bar{l}}$ susceptibilities almost coincide if the effective Polyakov loop potential is parameterized as in Eq. (5.4). The phase diagram calculated with an improved potential, shown in Fig. 22, is similar to that obtained with the previous choice of the Polyakov loop interactions, Fig. 16. This is also the case for the suscep- 


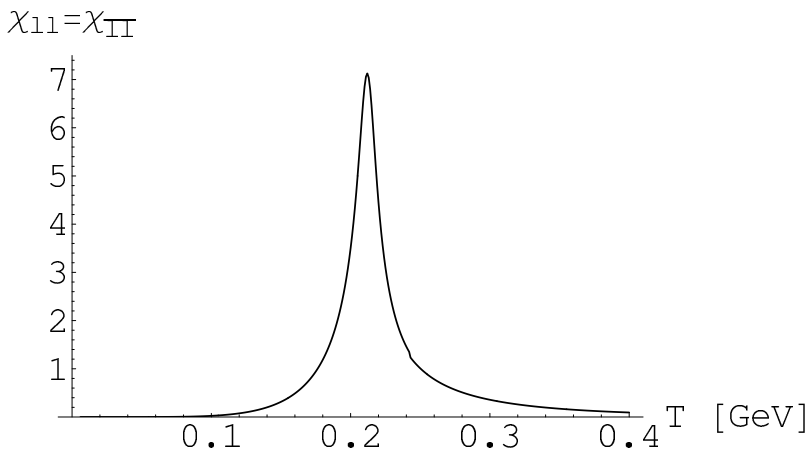

FIG. 21: The diagonal $\chi_{l l}=\chi_{\overline{l l}}$ susceptibility in the chiral limit as a function of temperature $T$ for $\mu=0$. The effective Polyakov loop potential (5.4) was used.

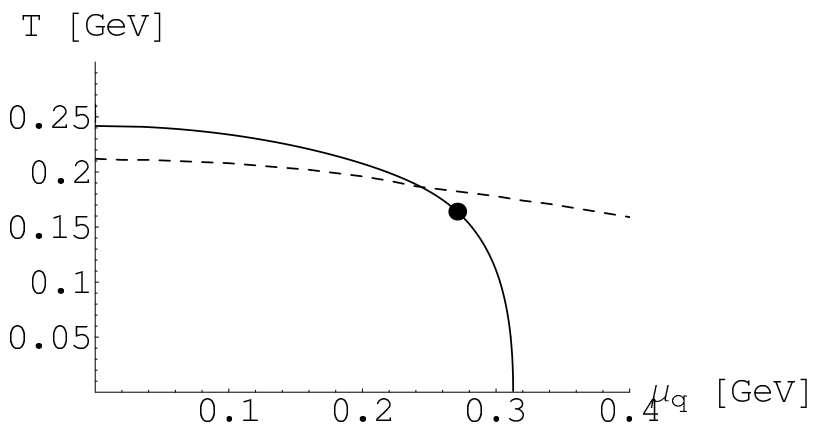

FIG. 22: The phase diagram of the PNJL model in the chiral limit. The solid (dashed) line denotes the chiral (deconfinement) phase transition respectively. The TCP (bold-point) is located at $\left(T_{c}=164, \mu_{c}=271\right) \mathrm{MeV}$. The parameter set (a) given in Table III was used in the calculations.

tibilities $\chi_{m m}$ and $\chi_{l \bar{l}}$ which have the same qualitative structure for both effective potentials.

\section{SUMMARY AND CONCLUSIONS}

We have explored the thermodynamical properties and the critical behavior of a system that exhibits an invariance under $Z(3)$ and chiral transformations. As a model we have use an extension of the NJL model for quarks with three colors and two flavors, which are coupled to an effective gluon field described by the Polyakov loop. In this model (the PNJL model) a non-local interaction instead of the point-like four-fermion couplings is employed. The PNJL model exhibits two essential features of QCD: a spontaneous chiral symmetry breaking and a "confinement" like property. Furthermore, due to the symmetries of the Lagrangian, the PNJL model belongs to the same universality class as that expected for QCD. Thus, such a model can be considered as a testing ground for the critical phenomena related to the breaking of the global $Z(3)$ and chiral symmetries.

Within the PNJL model, we discussed the phase dia- gram and the order of the phase transition, using mean field dynamics for different values of the parameters. The properties of thermodynamic quantities related with the quark degrees of freedom, like the quark number density and susceptibility, were analyzed in the vicinity of the chiral and deconfinement transitions.

We introduced susceptibilities related with the three different order parameters in this model, and analyzed their properties and their behavior near the phase transitions. We have shown that there are as many as nine susceptibilities that can be used to identify the phase structure of the model. In particular, for the quarkantiquark and chiral density-density correlations we have discussed the interplay between the restoration of chiral symmetry and deconfinement. We argued that in the actual formulation of the PNJL model a coincidence of the deconfinement and chiral symmetry restoration is accidental.

We found that, within the mean field approximation and with the present form of an effective gluon potential the correlations of the Polyakov loops in the quark-quark channel show an unphysical behavior, being negative in a broad parameter range. This behavior was traced back to the parameterization of the Polyakov loop potential. We argue that the $Z(N)$-invariance of this potential and the fit to lattice thermodynamics in the pure gluon sector is not sufficient to provide correct description of the Polyakov loop fluctuations in the presence of quarks in a medium. We note, however, that the improved potential of ref. [13] yields a positive, i.e. physical, $\chi_{l l}$, in qualitative agreement with the LGT results.

\section{Acknowledgments}

We acknowledge stimulating discussions with D. Blaschke, A. Dumitru, S. Ejiri, F. Karsch, H. Gies, J. Pawlowski, H. J. Pirner, C. Ratti, B. J. Schaefer, D. Voskresensky, J. Wambach and W. Weise. K.R. acknowledges fruitful discussion with R.D. Pisarski. The work of B.F. and C.S. was supported in part by the Virtual Institute of the Helmholtz Association under the grant No. VH-VI-041. K.R. acknowledges partial support of the Gesellschaft für Schwerionenforschung (GSI) and KBN under grant 2P03 (06925).

\section{APPENDIX A: FUNCTIONAL INTEGRAL AND PARTITION FUNCTION OF THE MODEL}

The Polyakov loop $L$ is a complex $3 \times 3$ matrix and can be diagonalized as in Eq. (2.7). Thus, the thermodynamic potential (2.15) is a functional of complex variables. However, as a physical observable the imaginary part of $\Omega$ must vanish. In this Appendix we show that this indeed happens. We follow the method that has been used in the context of a strong coupling QCD and the matrix model [38, 39]. 
We start with the partition function,

$$
Z=\int \mathcal{D} \psi \mathcal{D} \bar{\psi} \mathcal{D} L\left(\varphi, \varphi^{\prime}\right) e^{S[\psi, \bar{\psi}, L]},
$$

with the action $S$ being divided into three pieces:

$$
\begin{aligned}
S[\psi, \bar{\psi}, L] & =\int_{0}^{\beta} d \tau \int d^{3} x \mathcal{L}[\psi, \bar{\psi}, L] \\
& =S_{g}[L]+S_{q}[\psi, \bar{\psi}, L]+S_{\mathrm{int}}[\psi, \bar{\psi}] .
\end{aligned}
$$

It is quite transparent that the pure gluon part is real. Thus, we focus only on the quark part $S_{q}$. Performing the functional integral over fermion fields one gets,

$$
Z_{q}=\int \mathcal{D} L\left(\varphi, \varphi^{\prime}\right) \operatorname{Det} D\left(\varphi, \varphi^{\prime}\right),
$$

where $D$ denotes the Dirac operator

$$
D\left(\varphi, \varphi^{\prime}\right)=\not p-M-\gamma_{0}\left(\mu+i A_{4}\right) .
$$

Summing up the Matsubara frequencies, $p_{0}=i \omega_{n}=i(2 n+1) \pi T$ the partition function is obtained as

$$
\begin{aligned}
\ln Z_{q} & =\int \mathcal{D} L\left(\varphi, \varphi^{\prime}\right) \sum_{n} \int \frac{d^{3} p}{(2 \pi)^{3}} \operatorname{Tr} \ln D\left(\varphi, \varphi^{\prime}\right) \\
& =2 N_{f} \int \mathcal{D} L\left(\varphi, \varphi^{\prime}\right) \int \frac{d^{3} p}{(2 \pi)^{3}}\left[\operatorname{Tr}_{c} \ln \left(1+L\left(\varphi, \varphi^{\prime}\right) e^{-\beta E^{(+)}}\right)+\operatorname{Tr}_{c} \ln \left(1+L^{\dagger}\left(\varphi, \varphi^{\prime}\right) e^{-\beta E^{(-)}}\right)\right]
\end{aligned}
$$

taking the trace over color, flavor and Dirac variables in the above equation the imaginary part $I$ of $\ln Z_{q}$ is proportional to

$$
\begin{gathered}
I(M, \Phi, \bar{\Phi} ; T, \mu)=\int \mathcal{D} L\left(\varphi, \varphi^{\prime}\right) \int \frac{d^{3} p}{(2 \pi)^{3}}\left\{\tan ^{-1}\left[\frac{3 \operatorname{Im} \Phi\left(1-e^{-E^{(+)} / T}\right) e^{-E^{(+)} / T}}{1+3 \operatorname{Re} \Phi\left(1+e^{-E^{(+)} / T}\right) e^{-E^{(+)} / T}+e^{-3 E^{(+)} / T}}\right]\right. \\
\left.+\tan ^{-1}\left[\frac{-3 \operatorname{Im} \Phi\left(1-e^{-E^{(-)} / T}\right) e^{-E^{(-)} / T}}{1+3 \operatorname{Re} \Phi\left(1+e^{-E^{(-)} / T}\right) e^{-E^{(-)} / T}+e^{-3 E^{(-)} / T}}\right]\right\},
\end{gathered}
$$

where $\varphi$ and $\varphi^{\prime}$ dependence were suppressed and $\operatorname{Re} \Phi=\operatorname{Re} \bar{\Phi}$ and $\operatorname{Im} \Phi=-\operatorname{Im} \bar{\Phi}$ were used. Now let us replace the variables $\varphi, \varphi^{\prime}$ with $\varphi \rightarrow-\varphi$ and $\varphi^{\prime} \rightarrow-\varphi^{\prime}$. The $\mathrm{SU}(3)$ Haar measure $\mathcal{D} L\left(\varphi, \varphi^{\prime}\right)$ is unchanged under these replacements while $\operatorname{Im} \Phi$ changes its sign. Therefore, the first and second terms are separately odd under the change of group variables and vanish after the integration.

The thermal expectation values of complex $\Phi$ and $\bar{\Phi}$ are evaluated as

$$
\begin{aligned}
& \langle\Phi\rangle=\frac{1}{Z} \int \mathcal{D} L\left(\varphi, \varphi^{\prime}\right) e^{S_{g}+S_{\mathrm{int}}}\left(\operatorname{Re} z_{q} \cdot \operatorname{Re} \Phi-\operatorname{Im} z_{q} \cdot \operatorname{Im} \Phi\right) \\
& \langle\bar{\Phi}\rangle=\frac{1}{Z} \int \mathcal{D} L\left(\varphi, \varphi^{\prime}\right) e^{S_{g}+S_{\mathrm{int}}}\left(\operatorname{Re} z_{q} \cdot \operatorname{Re} \Phi+\operatorname{Im} z_{q} \cdot \operatorname{Im} \Phi\right)
\end{aligned}
$$

with

$$
Z=\int \mathcal{D} L\left(\varphi, \varphi^{\prime}\right) e^{S_{g}+S_{\mathrm{int}}}\left(\operatorname{Re} z_{q}+i \operatorname{Im} z_{q}\right)
$$

It is clear from Eq. A.6) that the imaginary part of the potential vanishes at $\mu=0$. Thus, the difference between $\langle\Phi\rangle$ and $\langle\bar{\Phi}\rangle$ comes only from the non-vanishing $\operatorname{Im} z_{q}$ at finite $\mu$. This can be also seen in the matrix model for color SU(3) symmetry [39]. 


\section{APPENDIX B: DERIVATIVES OF EFFECTIVE CONDENSATES}

In this appendix we summarize the derivatives of effective condensates with respect to $\mu$ and $T$. Taking the $\mu$-derivatives in the coupled gap equations (2.18)-(2.20) one gets

$$
\begin{aligned}
\frac{\partial M}{\partial \mu} & =\frac{T}{\Lambda}\left(A_{M}^{(\mu)} \chi_{m m}+\frac{T}{\Lambda} A_{\Phi}^{(\mu)} \chi_{m l}+\frac{T}{\Lambda} A_{\bar{\Phi}}^{(\mu)} \chi_{m \bar{l}}\right) \\
\frac{\partial \Phi}{\partial \mu} & =\frac{T}{\Lambda^{2}}\left(A_{M}^{(\mu)} \chi_{m l}+\frac{T}{\Lambda} A_{\Phi}^{(\mu)} \chi_{l l}+\frac{T}{\Lambda} A_{\bar{\Phi}}^{(\mu)} \chi_{l \bar{l}}\right), \\
\frac{\partial \bar{\Phi}}{\partial \mu} & =\frac{T}{\Lambda^{2}}\left(A_{M}^{(\mu)} \chi_{m \bar{l}}+\frac{T}{\Lambda} A_{\Phi}^{(\mu)} \chi_{l \bar{l}}+\frac{T}{\Lambda} A_{\bar{\Phi}}^{(\mu)} \chi_{\overline{l l}}\right),
\end{aligned}
$$

where $\chi_{i j}$ are defined in Section 4 and the functions $A^{(\mu)}$ are introduced as

$$
\begin{aligned}
A_{M}^{(\mu)}= & -\frac{6 N_{f}}{T^{3}} \int \frac{d^{3} p}{(2 \pi)^{3}} \frac{M_{p} f^{2}(p)}{E_{p}}\left[\frac { e ^ { - E ^ { ( + ) } / T } } { ( g ^ { ( + ) } ) ^ { 2 } } \left\{\Phi+4 \bar{\Phi} e^{-E^{(+)} / T}+3(1+\bar{\Phi} \Phi) e^{-2 E^{(+)} / T}\right.\right. \\
& \left.\left.+4 \Phi e^{-3 E^{(+)} / T}+\bar{\Phi} e^{-4 E^{(+)} / T}\right\}-(\bar{\Phi}, \Phi ;-\mu)\right] \\
A_{\Phi}^{(\mu)}= & \frac{6 N_{f}}{T^{3}} \int \frac{d^{3} p}{(2 \pi)^{3}}\left[\frac{e^{-E^{(+)} / T}}{\left(g^{(+)}\right)^{2}}\left\{1-3 \bar{\Phi} e^{-2 E^{(+)} / T}-2 e^{-3 E^{(+)} / T}\right\}\right. \\
& \left.-\frac{e^{-2 E^{(-)} / T}}{\left(g^{(-)}\right)^{2}}\left\{2+3 \bar{\Phi} e^{-E^{(-)} / T}-e^{-3 E^{(-)} / T}\right\}\right] \\
A_{\bar{\Phi}}^{(\mu)}= & A_{\Phi}^{(\mu)}(\bar{\Phi}, \Phi ;-\mu) .
\end{aligned}
$$

The required temperature derivatives of order parameters are directly obtained from the gap equations as

$$
\begin{aligned}
\frac{\partial M}{\partial T} & =\frac{T}{\Lambda}\left(A_{M}^{(T)} \chi_{m m}+\frac{T}{\Lambda} A_{\Phi}^{(T)} \chi_{m l}+\frac{T}{\Lambda} A_{\bar{\Phi}}^{(T)} \chi_{m \bar{l}}\right) \\
\frac{\partial \Phi}{\partial T} & =\frac{T}{\Lambda^{2}}\left(A_{M}^{(T)} \chi_{m l}+\frac{T}{\Lambda} A_{\Phi}^{(T)} \chi_{l l}+\frac{T}{\Lambda} A_{\bar{\Phi}}^{(T)} \chi_{l \bar{l}}\right) \\
\frac{\partial \bar{\Phi}}{\partial T} & =\frac{T}{\Lambda^{2}}\left(A_{M}^{(T)} \chi_{m \bar{l}}+\frac{T}{\Lambda} A_{\Phi}^{(T)} \chi_{l \bar{l}}+\frac{T}{\Lambda} A_{\bar{\Phi}}^{(T)} \chi_{\overline{l l}}\right)
\end{aligned}
$$

where the functions $A^{(T)}$ are defined as

$$
\begin{aligned}
A_{M}^{(T)}= & -\frac{6 N_{f}}{T^{4}} \int \frac{d^{3} p}{(2 \pi)^{3}} \frac{M_{p} f^{2}(p)}{E_{p}}\left[\frac { e ^ { - E ^ { ( + ) } / T } } { ( g ^ { ( + ) } ) ^ { 2 } } E ^ { ( + ) } \left\{\Phi+4 \bar{\Phi} e^{-E^{(+)} / T}+3(1+\bar{\Phi} \Phi) e^{-2 E^{(+)} / T}\right.\right. \\
& \left.\left.+4 \Phi e^{-3 E^{(+)} / T}+\bar{\Phi} e^{-4 E^{(+)} / T}\right\}+(\bar{\Phi}, \Phi ;-\mu)\right] \\
A_{\Phi}^{(T)}= & \frac{T}{2} \frac{\partial b_{2}}{\partial T} \bar{\Phi}+\frac{6 N_{f}}{T^{4}} \int \frac{d^{3} p}{(2 \pi)^{3}}\left[\frac{e^{-E^{(+)} / T}}{\left(g^{(+)}\right)^{2}} E^{(+)}\left\{1-3 \bar{\Phi} e^{-2 E^{(+)} / T}-2 e^{-3 E^{(+)} / T}\right\}\right. \\
& \left.-\frac{e^{-2 E^{(-)} / T}}{\left(g^{(-)}\right)^{2}} E^{(-)}\left\{2+3 \bar{\Phi} e^{-E^{(-)} / T}-e^{-3 E^{(-)} / T}\right\}\right]-\frac{18 N_{f}}{T^{3}} \int \frac{d^{3} p}{(2 \pi)^{3}}\left[\frac{e^{-E^{(+)} / T}}{g^{(+)}}+\frac{e^{-2 E^{(-)} / T}}{g^{(-)}}\right] \\
A_{\bar{\Phi}}^{(T)}= & A_{\Phi}^{(T)}(\bar{\Phi}, \Phi ;-\mu) .
\end{aligned}
$$

\section{APPENDIX C: PARAMETERS USED IN THE PNJL MODEL THERMODYNAMICS}

The compilation of parameters used in the model calculations is given in the following tables: 


\begin{tabular}{c|ccc}
\hline input & $f_{\pi}=92.4 \mathrm{MeV}$ & $m_{\pi}=135 \mathrm{MeV}$ & $M=335 \mathrm{MeV}$ \\
\hline \hline non-local NJL model & $\Lambda=684.2 \mathrm{MeV}$ & $G_{S} \Lambda^{2}=4.66$ & $m_{0}=4.46 \mathrm{MeV}$ \\
\hline local NJL model & $\Lambda=625.1 \mathrm{MeV}$ & $G_{S} \Lambda^{2}=4.38$ & $m_{0}=5.31 \mathrm{MeV}$ \\
\hline
\end{tabular}

TABLE I: Set of parameters for the NJL sector [19]. The parameters of non-local NJL model were fixed for $\alpha=10$.

\begin{tabular}{cccccc}
\hline$a_{0}$ & $a_{1}$ & $a_{2}$ & $a_{3}$ & $b_{3}$ & $b_{4}$ \\
\hline 6.75 & -1.95 & 2.625 & -7.44 & 0.75 & 7.5 \\
\hline
\end{tabular}

TABLE II: Set of parameters for the Polyakov-loop effective potential [5].

[1] A. Gocksch and M. Ogilvie, Phys. Rev. D 31, 877 (1985).

[2] P. N. Meisinger and M. C. Ogilvie, Phys. Lett. B 379, 163 (1996); P. N. Meisinger, T. R. Miller and M. C. Ogilvie, Phys. Rev. D 65, 034009 (2002).

[3] K. Fukushima, Phys. Lett. B 591, 277 (2004).

[4] F. Sannino, Phys. Rev. D 66, 034013 (2002); A. Mocsy, F. Sannino and K. Tuominen, Phys. Rev. Lett. 92, 182302 (2004).

[5] C. Ratti, M. A. Thaler and W. Weise, Phys. Rev. D 73, 014019 (2006).

[6] S. Digal, E. Laermann and H. Satz, Eur. Phys. J. C 18, 583 (2001).

[7] E. Megias, E. Ruiz Arriola and L. L. Salcedo, Phys. Rev. D 74, 065005 (2006); arXiv:hep-ph/0607338

[8] E. M. Ilgenfritz and J. Kripfganz, Z. Phys. C 29, 79 (1985).

[9] K. Fukushima, Phys. Lett. B 553, 38 (2003); Phys. Rev. D 68, 045004 (2003).

[10] Y. Nambu and G. Jona-Lasinio, Phys. Rev. 122, 345 (1961); Phys. Rev. 124, 246 (1961).

[11] For reviews and applications of the NJL model to hadron physics, see e.g., U. Vogl and W. Weise, Prog. Part. Nucl. Phys. 27, 195 (1991); S. P. Klevansky, Rev. Mod. Phys. 64 (1992) 649; T. Hatsuda and T. Kunihiro, Phys. Rept. 247, 221 (1994); M. Buballa, Phys. Rept. 407, 205 (2005).

[12] S. K. Ghosh, T. K. Mukherjee, M. G. Mustafa and R. Ray, Phys. Rev. D 73, 114007 (2006).

[13] S. Roessner, C. Ratti and W. Weise, arXiv:hep-ph/0609281.

[14] S. Mukherjee, M. G. Mustafa and R. Ray, arXiv:hep-ph/0609249.

[15] H. Hansen, W. M. Alberico, A. Beraudo, A. Molinari, M. Nardi and C. Ratti, arXiv:hep-ph/0609116.

[16] R. D. Pisarski, Phys. Rev. D 62, 111501 (2000); A. Dumitru and R. D. Pisarski, Phys. Lett. B 504, 282 (2001); Phys. Lett. B 525, 95 (2002).

[17] Z. Zhang and Y. X. Liu, arXiv:hep-ph/0610221.

[18] S. M. Schmidt, D. Blaschke and Y. L. Kalinovsky, Phys. Rev. C 50, 435 (1994).

[19] H. Grigorian, arXiv:hep-ph/0602238

[20] L. D. McLerran and B. Svetitsky, Phys. Lett. B 98, 195 (1981); Phys. Rev. D 24, 450 (1981).

[21] R. D. Pisarski and F. Wilczek, Phys. Rev. D 29, 338 (1984).
[22] Y. Hatta and T. Ikeda, Phys. Rev. D 67, 014028 (2003).

[23] H. Fujii, Phys. Rev. D 67, 094018 (2003); H. Fujii and M. Ohtani, Phys. Rev. D 70, 014016 (2004).

[24] M. A. Stephanov, Prog. Theor. Phys. Suppl. 153, 139 (2004) [Int. J. Mod. Phys. A 20, 4387 (2005)].

[25] K. Redlich, B. Friman and C. Sasaki, J. Phys. G 32, S283 (2006).

[26] C. Sasaki, B. Friman and K. Redlich, arXiv:hep-ph/0611143

[27] C. R. Allton, M. Doring, S. Ejiri, S.J. Hands, O. Kaczmarek, F. Karsch, E. Laermann and K. Redlich, Phys. Rev. D 71, 054508 (2005).

[28] L.D. Landau and E.M. Lifshitz, Statistical Physics, Course of Theoretical Physics, Vol. 5, (Pergamon Press, New York, 1980) Chapter XIV

[29] O. Kaczmarek and F. Zantow, Phys. Rev. D 71, 114510 (2005)

[30] Z. Fodor and S. D. Katz, JHEP 0203, 014 (2002); C. R. Allton, S. Ejiri, S. J. Hands, O. Kaczmarek, F. Karsch, E. Laermann and C. Schmidt, Phys. Rev. D 68, 014507 (2003); M. D'Elia and M. P. Lombardo, Phys. Rev. D 67, 014505 (2003); P. de Forcrand and O. Philipsen, Nucl. Phys. B 673, 170 (2003)

[31] F. Karsch and E. Laermann, Phys. Rev. D 50, 6954 (1994); C. R. Allton et al., Phys. Rev. D 66, 074507 (2002).

[32] M. Cheng et al., Phys. Rev. D 74, 054507 (2006) arXiv:hep-lat/0608013.

[33] E. V. Shuryak, Phys. Lett. B 107, 103 (1981).

[34] R. D. Pisarski, Phys. Lett. B 110, 155 (1982).

[35] J. Pawlowski, B.-J. Schaefer and J. Wambach, to be published

[36] A. Dumitru and R. D. Pisarski, Phys. Rev. D 66, 096003 (2002).

[37] S. Nadkarni, Phys. Rev. D 33, 3738 (1986).

[38] D. E. Miller and K. Redlich, Phys. Rev. D 37, 3716 (1988); Phys. Rev. D 35, 2524 (1987). K. Redlich, F. Karsch and A. Tounsi, in proceedings of "24th International Colloquium on Group Theoretical Methods in Physics: GROUP - 24: (ICGTMP 2002)", Published in *Paris 2002, Physical and mathematical aspects of symmetries*; hep-ph/0302245

[39] A. Dumitru, R. D. Pisarski and D. Zschiesche, Phys. Rev. D 72, 065008 (2005). A. Dumitru, Y. Hatta, J. Lenaghan, K. Orginos and R. D. Pisarski, Phys. Rev. D 70, 034511 


\begin{tabular}{l||l}
\hline set (a) & set (b) \\
\hline$\Lambda=684.2 \mathrm{MeV}$ & $\Lambda=684.2 \mathrm{MeV}$ \\
$G_{S} \Lambda^{2}=4.66$ & $G_{S} \Lambda^{2}=4.05$ \\
$T_{0}=270 \mathrm{MeV}$ & $T_{0}=225 \mathrm{MeV}$ \\
\hline$T_{\text {ch }}(\mu=0)=242 \mathrm{MeV}$ & $T_{\text {ch }}(\mu=0)=180 \mathrm{MeV}$ \\
$T_{\text {dec }}(\mu=0)=217 \mathrm{MeV}$ & $T_{\text {dec }}(\mu=0)=180 \mathrm{MeV}$ \\
\hline
\end{tabular}

TABLE III: Set of parameters in the chiral limit used in this work and the resultant phase transition temperatures. $\alpha$ was fixed to be $\alpha=10$.

\begin{tabular}{cccc}
\hline$a_{0}$ & $a_{1}$ & $a_{2}$ & $b_{3}$ \\
\hline 3.51 & -2.47 & 15.22 & -1.75 \\
\hline
\end{tabular}

TABLE IV: Set of parameters for the improved Polyakov-loop effective potential [13].

(2004). 